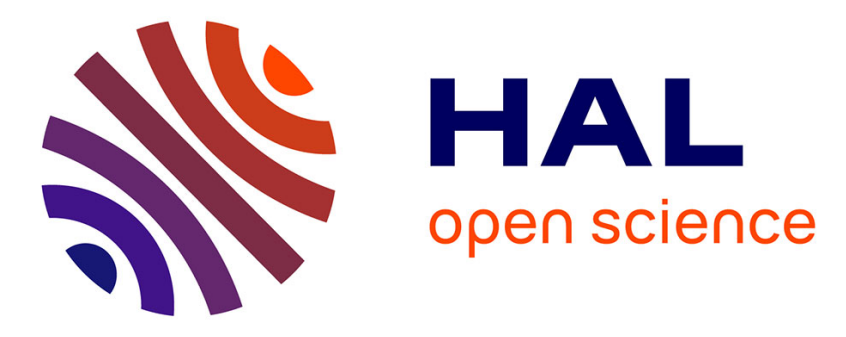

\title{
Dynamics of inverted flags: Experiments and comparison with theory
}

\author{
Mohammad Tavallaeinejad, Manuel Flores Salinas, Michael Païdoussis, \\ Mathias Legrand, Mojtaba Kheiri, Ruxandra Botez
}

\section{To cite this version:}

Mohammad Tavallaeinejad, Manuel Flores Salinas, Michael Païdoussis, Mathias Legrand, Mojtaba Kheiri, et al.. Dynamics of inverted flags: Experiments and comparison with theory. Journal of Fluids and Structures, 2021, 101, pp.103199. 10.1016/j.jfluidstructs.2020.103199 hal-03124681

\section{HAL Id: hal-03124681 \\ https://hal.science/hal-03124681}

Submitted on 5 Feb 2021

HAL is a multi-disciplinary open access archive for the deposit and dissemination of scientific research documents, whether they are published or not. The documents may come from teaching and research institutions in France or abroad, or from public or private research centers.
L'archive ouverte pluridisciplinaire HAL, est destinée au dépôt et à la diffusion de documents scientifiques de niveau recherche, publiés ou non, émanant des établissements d'enseignement et de recherche français ou étrangers, des laboratoires publics ou privés. 


\title{
Dynamics of inverted flags: experiments and comparison with theory
}

\author{
Mohammad Tavallaeinejad $^{\mathrm{a}}$, Manuel Flores Salinas ${ }^{\mathrm{b}}$, Michael P. Païdoussis ${ }^{\mathrm{a}}$, Mathias Legrand ${ }^{\mathrm{a}}$, Mojtaba \\ Kheiric $^{\mathrm{c}}$, Ruxandra M. Botez ${ }^{\mathrm{b}}$

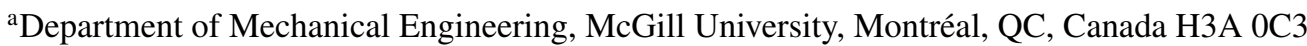 \\ ${ }^{\mathrm{b}}$ Le Laboratoire de recherche en commande active, avionique et aéroservoélasticité (LARCASE), École de \\ Technologie Supérieure, Montréal, QC, Canada, H3C 6M8 \\ ${ }^{\mathrm{c}}$ Department of Mechanical, Industrial and Aerospace Engineering, Concordia University, Montréal, QC, \\ Canada, H3G 1M8
}

\begin{abstract}
The study of the dynamics of cantilevered thin flexible plates in reverse axial flow — also known as inverted flags - has become of significant interest, partly due to their energy harvesting potential. This paper presents fresh experimental results, aiming to enhance our understanding of the dynamics of inverted flags, particularly concerning stability and global dynamics sensitivity to various system parameters, such as the aspect ratio (i.e. height-to-length ratio) and flag material. This is achieved through testing flags of various dimensions, made from different materials. The dynamics of the system is presented in the form of bifurcation diagrams in which the flag tip angle is shown as a function of the dimensionless flow velocity. The frequency content of oscillatory motions is presented in the form of spectrograms. Also, time-histories, phase-plane portraits, as well as power spectral density and probability density function plots are presented for different flow regimes. Interesting dynamical features, such as small- and large-amplitude flapping, static divergence, large-amplitude buckling, and chaotic motions, have been observed experimentally, some for the first time. It is shown that for flags of very low aspect ratio the undeflected static equilibrium is stable prior to a subcritical pitchfork bifurcation. For flags of large aspect ratio, on the other hand, the undeflected static equilibrium becomes unstable via a supercritical pitchfork bifurcation leading to static divergence (buckling) at a sufficiently high flow velocity; at higher flow velocities, past the pitchfork bifurcation, a supercritical Hopf bifurcation materialises leading to flapping motion around the deflected static equilibrium; at even higher flow velocities, flapping motion becomes symmetric around the undeflected static equilibrium. Interestingly, it is found that heavy flags may exhibit large-amplitude flapping right after the initial static equilibrium, provided that they are subjected to a sufficiently large disturbance. In addition to experiments, nonlinear analytical models for the two asymptotic cases of zero and infinite aspect ratios are outlined, the results of which are used for comparison with the experimental results. Theory and experiments are found to be in reasonably good agreement.
\end{abstract}

Keywords inverted flag, cantilevered plates in reverse axial flow, fluid-structure interactions, nonlinear dynamics, effect of aspect ratio, effect of flag material

\section{Introduction}

Experimental observations found in the literature suggest that the inverted-flag system may display a wide range of dynamical behaviour depending on the system parameters, such as (i) the fluid-to-plate mass ratio $\mu=\rho_{\mathrm{f}} L / \rho_{\mathrm{p}} h ;^{1} \rho_{\mathrm{f}}$ and $\rho_{\mathrm{p}}$ being the mass density of the fluid and plate, respectively, and $h$ and $L$ the thickness and the length of the plate, respectively, (ii) the flag aspect (height-to-length) ratio $A=H / L ; H$ being the height of the plate, and (iii) the initial angle of attack. Inverted flags with a sufficiently large aspect ratio (i.e., $R>0.1$ ) may exhibit: (a) a stretched-straight state at low flow velocities, characterised by the plate resting at its initial configuration, (b) a large-amplitude flapping state at sufficiently high flows, and (c) a fully-bent state, where the plate stops flapping and bends backwards away from the initial static equilibrium. In this paper, we refer to this class as large-aspect-ratio inverted flags. On the other hand, very low aspect ratio (slender) inverted flags (i.e., $R \lesssim 0.1$ ) may undergo static divergence only through a subcritical pitchfork bifurcation, i.e. going directly from (a) to (c) and via a different route.

The study of the dynamics of inverted flags has become of significant interest in recent years, not only as a "curiosity-driven" research because of the rich dynamics they display, but also because "inverted flags" are present in several engineering applications and biological systems. Examples are small-scale energy harvesting systems (Orrego et al. 2017), vertical-axis wind turbines with pliable blades (Cossé 2014), heat transfer enhancement elements in heat exchangers (Park et al. 2016; Chen et al. 2018; Li et al. 2019), mixing enhancement elements (Yu et al. 2019), and devices for wake sensing purposes in small unmanned aerial vehicles (Gunasekaran et al. 2019).

\footnotetext{
${ }^{1}$ In the present paper, including the Introduction where various existing works are discussed, the mass ratio is defined as fluid/structure; it is noted, however, that the inverse definition, structure/fluid, is utilized in some other papers.
} 
Shoele \& Mittal (2016), for example, explored the energy harvesting efficiency of an inverted piezoelectric flag. They showed that the unique properties of the inverted-flag system make it an ideal candidate for small-scale energy harvesting applications, outperforming conventional piezoelectric flags with a clamped leading edge and a free trailing edge. In particular, an inverted flag may undergo large-amplitude flapping oscillations, resulting in a larger bending strain energy and superior energy harvesting performance compared to conventional flags (e.g., refer to Michelin \& Doaré 2013). As another potential application, Park et al. (2016) proposed that utilizing a self-oscillating inverted-flag significantly enhanced the heat transfer and led to a significant increase in the thermal efficiency factor, much larger than that obtained using a conventional flag (Lee et al. 2018).

Unlike the conventional flag configuration, few experiments have previously been conducted on inverted configurations, such as the so-called clapping book (i.e. a stack of small-aspect-ratio elastic sheets in wind) by Buchak et al. (2010), free-clamped cylinders (i.e. cylinders clamped at the trailing edge and free at the leading one) subjected to axial air-flow by Rinaldi \& Païdoussis $(2012,2020)$, as well as in water flow by the first author of the present paper; also, with inverted flags in air-flow by the Gharib group at Caltech (Kim et al. 2013, Sader et al. 2016a, Cossé et al. 2014, Sader et al. 2016b and Fan et al. 2019) or in water flow (Kim et al. 2013 and Yu et al. 2017).

The purpose of this paper is to establish a comprehensive picture of the global dynamics of inverted flags and provide new insight into the flapping mechanism. Some experimental results previously obtained by others are reproduced, by covering a similar range of system parameters while using a low-turbulence wind tunnel with a large test-section and different measurement techniques, to increase reliability of findings and to ensure their reproducibility. New insights on the global dynamics of large aspect-ratio flags are provided via constructing bifurcation diagrams for the tip ${ }^{2}$ rotation angle of the flags. Utilizing various tools and techniques, such as power density function diagrams, spectrograms, and phase-plane portraits, extensive investigations are conducted into the dynamical aspects of each flow regime. For the first time, the onset of divergence of large aspect-ratio inverted flags is pinpointed experimentally. Moreover, experiments are conducted with flags of different materials, providing new insights into the effects of material on the dynamics. Finally, the recently developed theoretical models are next validated by comparison with the new experimental results. The system of interest is defined in the theoretical modelling section of the paper, which is presented ahead of the experimental part, together with some typical theoretical results.

To this end, in Section 2, analytical representations of the fluid forces acting on the inverted flag are employed in the two asymptotic aspect ratio limits. Concerning the solution technique, effective numerical frameworks are implemented to generate results. Experiments on inverted flags in axial flow are described in Section 3, with the flag at rest in a vertical plane. The experiments were carried out in a subsonic wind tunnel in order to systematically investigate the system behaviour, both the onset of instability and the post-critical dynamics, for various sets of system parameters. Finally, in Section 8 the reliability of analytical tools to investigate the behaviour of inverted flags is demonstrated.

\section{Theoretical modelling}

To construct an analytical model for the dynamics of inverted flags requires developing models for both the structural dynamics and the aerodynamics. The flapping motion of the inverted flag obeys the Euler-Bernoulli beam equation subjected to pressure forces which are calculated by a fluid dynamics model. Theoretical fluid dynamics models can be divided into two limiting categories according to the aspect ratio of the elastic member (i.e. width-to-length ratio). For asymptotically small aspect ratios, where the length of the flag is assumed to be infinite, the fluid forces can be calculated using the large-amplitude elongated (slender) body theory of Lighthill (1971). For infinite aspect ratios, where the width of the flag is assumed to be much larger than its length, a two-dimensional flow theory is relevant, as the flow around the flag may be modelled using Theodorsen's unsteady aerofoil theory (Theodorsen 1949).

The theoretical models are presented here in highly abbreviated form, as they have already been presented in detail elsewhere (Tavallaeinejad et al. 2018; 2020a; 2020b).

\subsection{Equation of motion}

The nonlinear governing equation of motion expressed in terms of the rotation angle $\psi(x, t)$ of the crosssection of a flag with width $H$, chord $L$, thickness $h$, cross-sectional area $A_{\mathrm{c}}=H h$, transverse moment of inertia $I$, mass density $\rho_{\mathrm{p}}$, Young's modulus $E$, flexural rigidity $D$, internal damping coefficient $\eta_{\mathrm{p}}$, and

\footnotetext{
${ }^{2}$ In this paper, tip refers to the free end or the leading edge of the inverted flag.
} 
Poisson ratio $v_{\mathrm{p}}$, subjected to a distributed force, $F_{\mathrm{N}}(x, t)$ can be derived as (cf. Tavallaeinejad et al. (2020b))

$$
\begin{aligned}
& \rho_{\mathrm{p}} I \ddot{\psi}(x, t)-D \partial_{x x} \psi(x, t)-D \eta_{\mathrm{p}} \dot{\psi}(x, t) \\
& +\rho_{\mathrm{p}} A_{\mathrm{c}}\left[\cos \psi(x, t) \partial_{t t}\left(\int_{x}^{L} \int_{0}^{s} \sin \psi(\xi, t) \mathrm{d} \xi \mathrm{d} s\right)-\sin \psi(x, t) \partial_{t t}\left(\int_{x}^{L} \int_{0}^{s} \cos \psi(\xi, t) \mathrm{d} \xi \mathrm{d} s\right)\right] \\
& +\cos \psi(x, t) \int_{L}^{x} F_{\mathrm{N}}(s, t) \cos \psi(s, t) \mathrm{d} s+\sin \psi(x, t) \int_{L}^{x} F_{\mathrm{N}}(s, t) \sin \psi(s, t) \mathrm{d} s=0,
\end{aligned}
$$

along with the associated clamped-free boundary conditions

$$
\psi(x=0, t)=0 \quad \text { and } \quad \partial_{x} \psi(x=L, t)=0,
$$

where $D=E I /\left(1-v_{\mathrm{p}}^{2}\right)$ is the plane-strain flexural rigidity for flags of large aspect ratios; in the case of a slender beam, the flexural rigidity becomes $D=E I ; I=H h^{3} / 12$.

Equation (1) describes the nonlinear, fully coupled, fluid-structure interaction. The control parameters are reduced to five dimensionless numbers defined as

$$
\begin{aligned}
& \mu=\frac{\rho_{\mathrm{f}} L}{\rho_{\mathrm{p}} h}: \text { mass ratio, } \\
& R=\frac{H}{L}: \text { aspect ratio, } \\
& \Pi=\sqrt{\frac{\rho_{\mathrm{f}} H L}{D}} L U: \text { dimensionless flow velocity, } \\
& f^{*}=f L^{2} \sqrt{\frac{\rho_{\mathrm{p}} A_{\mathrm{c}}}{D}}: \text { dimensionless frequency, } \\
& f_{\mathrm{R}}=\frac{f L}{U}=\frac{f^{*} \sqrt{\mu}}{\Pi}: \text { reduced frequency, } \\
& \operatorname{Re}_{\mathrm{L}}=\frac{U L}{v}: \text { Reynolds number, }
\end{aligned}
$$

where $v$ denotes the kinematic viscosity of the fluid.

In equation (1), $F_{\mathrm{N}}(x, t)$ represents the fluid-related forces, which may be determined for an inverted flag with low aspect ratio via slender-body theory. It may also be formulated for an infinitely wide inverted flag based on a two-dimensional flow model.

\subsection{Slender-body nonlinear model}

The stability and dynamics of low-aspect-ratio (slender) inverted flags has been studied first by Sader et al. (2016b) for a slender inverted flag in the asymptotic limit of zero aspect ratio, and extended by Tavallaeinejad et al. (2018) to finite aspect ratios and by Tavallaeinejad et al. (2020a) to unsteady flows. The effect of initial (residual) curvature was also studied by Tavallaeinejad et al. (2018). More specifically, Tavallaeinejad et al. $(2018,2020 a)$ utilized a continuum representation of fluid flow forces on the basis of the large amplitude elongated body theory of Lighthill (1971) to formulate the reactive force and the model of Bollay (1939) to express the nonlinear vortex lift mechanism associated with flow separation from the side edges.

Following the model developed by Tavallaeinejad et al. (2018) and using a pseudo-arclength continuation technique, the response of inverted flags with $R=0.1$ and $\mu=0.2$ is presented in Figure 1 in the from of bifurcation diagrams. Two cases are shown: one for a perfectly straight flag, and the other for a flag with an imperfection in the form of an initial curvature with a continuous, monotonically increasing deflection along the length of the flag, and maximum amplitude at the tip $\Delta / L=0.02$.

The bifurcation diagram for the perfectly straight inverted flag forms a strongly subcritical pitchfork bifurcation (divergence) at $\Pi_{\mathrm{P}}=5.81$ and a saddle-node bifurcation at $\Pi_{\mathrm{S}}=2.95$ (note the symmetrical response of the system with respect to the horizontal axis). On the other hand, for the flag with a small initial curvature, the pitchfork bifurcation is destroyed and is replaced by a saddle-node bifurcation. Hence, the response of the inverted flag displays a continuous deformation path incorporating two saddle-node bifurcations at $\Pi_{\mathrm{S} 1}=4.20$ and $\Pi_{\mathrm{S}}=2.91$.

For the stretched-straight flag, a bistable region can be identified in the range of flow velocity $\Pi=$ [2.95 5.81], wherein the inverted flag may undergo a jump from the undeflected equilibrium to the stable deflected equilibrium as a result of small perturbations arising from fluctuations in the impinging flow. According to the more realistic model with an initial curvature, the range of the bistable region becomes significantly smaller; consequently, the inverted flag may jump to the deflected equilibrium only at considerably smaller flow velocities. 


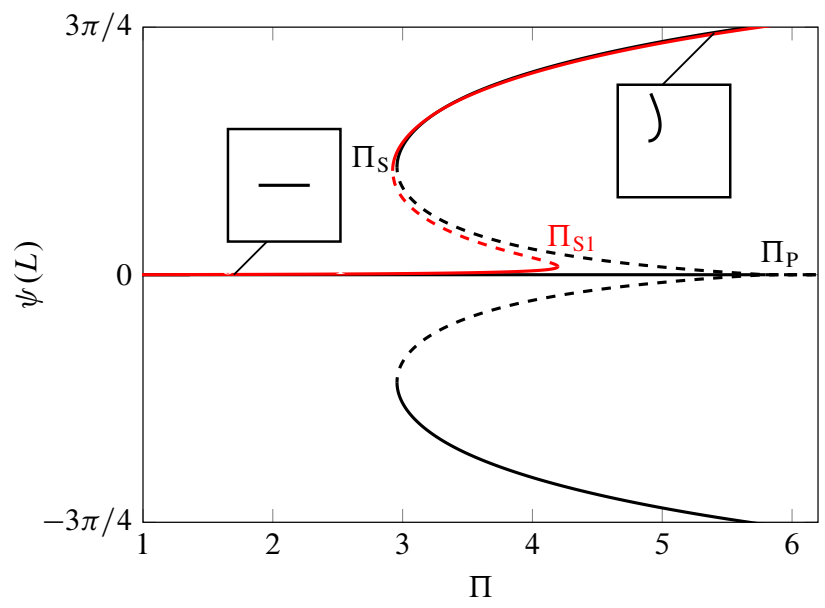

Figure 1: Bifurcation diagram for an inverted slender flag with $\mu=0.2$ and $R=0.1$; [ $[$ ] perfectly straight flag, [-] with small tip deflection $\Delta / L=0.02$; solid and dashed lines represent stable and unstable static solutions, respectively.

\subsection{Two-dimensional nonlinear model}

It has been shown by Sader et al. $(2016 a, b)$; Tavallaeinejad et al. $(2018,2020 b)$ that sufficiently wide flags and their slender counterparts exhibit different physics. The main difference is that for the wider flags separation occurs at the fore/aft edges, rather than the side edges of the flag.

Tavallaeinejad et al. (2020b) developed a nonlinear analytical model for the dynamics of inverted flags of high-aspect ratios. The quasi-steady version of Theodorsen's unsteady aerodynamic theory (Theodorsen 1949) is used for inviscid fluid-dynamic modelling of the deforming flag in axial flow, along with the Polhamus leading edge suction analogy (Polhamus 1966) to model flow separation effects from the free end of the flags at moderate angles of attack.

The bifurcation diagram for a two-dimensional inverted flag with $\mu=0.2$, perfectly aligned with the incident flow is shown in Figure 2. The numerical results show that the undeflected static equilibrium,

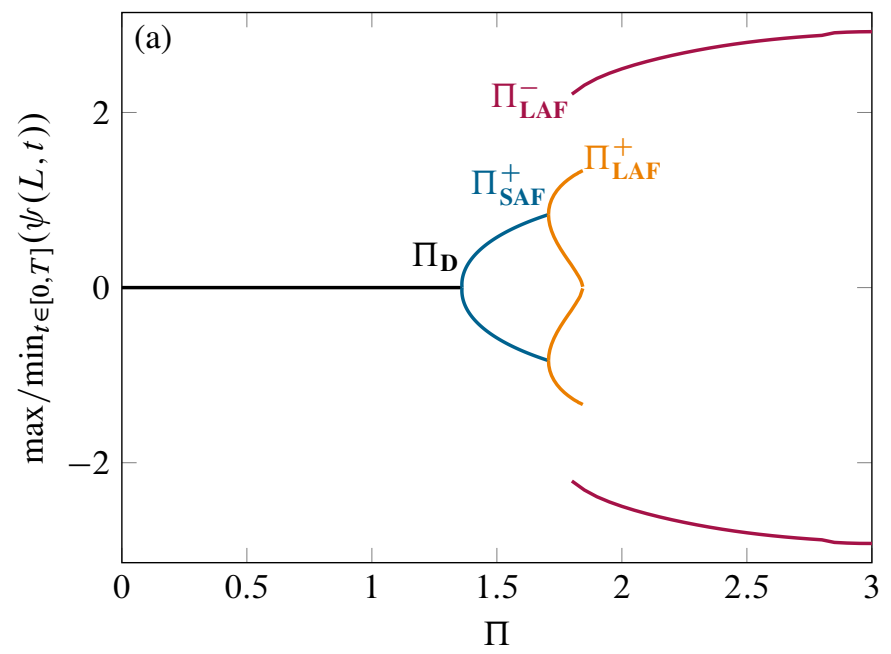

(b)

(c)

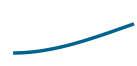

(d)

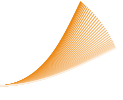

(e)

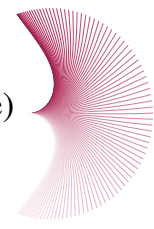

Figure 2: (a) Bifurcation diagram for the investigated two-dimensional inverted flag with $\mu=0.2$, indicating the peak values of the leading edge rotation over a cycle of steady-state oscillation of period $T^{*}$. [ - ] Stable static solution (undeflected stable equilibrium); [-] stable static solutions (deflected static equilibria) corresponding to buckling of the inverted flag to either side; $[-]$ stable periodic solutions (oscillation around deflected equilibria) corresponding to deformed-flapping of the inverted flag to either side; [-] stable periodic solution (oscillations around undeflected equilibrium). Shapes of the flag corresponding to each regime: (b) stretched-straight state ( $\Pi=1.35)$, (c) buckled state $(\Pi=1.40)$, (d) deformed-flapping state $(\Pi=1.75)$, and (e) large-amplitude flapping $(\Pi=1.65)$.

Figure 2(b), is stable at small flow velocities, and loses stability at $\Pi_{D}=1.36$ through a supercritical pitchfork bifurcation. This leads to a divergence (buckling) in the first mode, the amplitude of which increases with flow velocity (see Figure 2(c)). With increasing flow velocity, the deflected stable equilibrium loses stability via a supercritical Hopf bifurcation at $\Pi_{\mathrm{SAF}}^{+}=1.71$, leading to a periodic oscillation around the buckled state, as shown in Figure 2(d). Finally, at higher flow velocities, a large-amplitude flapping regime emerges 
from a saddle-node bifurcation at $\Pi_{\mathrm{LAF}}^{+}=1.85$. Decreasing the flow speed leads to a transition from the large-amplitude to the deformed-flapping regime at $\Pi_{\mathrm{LAF}}^{-}=1.80$. The notation $\Pi^{+}$and $\Pi^{-}$is used for dimensionless critical flow velocities obtained via sweeping up and down, respectively.

\section{Experimental methods}

\subsection{Experimental apparatus}

The experimental investigation was undertaken in the Aeronautical Research Laboratory in Active Control, Avionics and Aeroservoelasticity (LARCASE) of École de technologie supérieure (ÉTS), using the PricePaïdoussis subsonic blow-down wind tunnel. The test-section dimensions are $62 \mathrm{~cm} \times 91 \mathrm{~cm} \times 183 \mathrm{~cm}$ (height $\times$ width $\times$ length), and the maximum speed achievable in the test-section is about $30 \mathrm{~m} / \mathrm{s}$. Plexiglas windows on the test-section allow for camera-based motion capture as the main form of data acquisition, as shown in Figure 3. The turbulence intensity of the flow in the test-section is less than $1 \%$ (see Mosbah et al. 2013; Koreanschi et al. 2016), that is one order-of-magnitude smaller than that of the tunnel used by Kim et al. (2013) and Sader et al. $(2016 a, b)$, which was $\sim 9 \%$. The maximum blockage ratio (for a flag with $60 \mathrm{~cm} \times 15 \mathrm{~cm}$ ) is less that $30 \%$. In the present experiments, flags of various dimensions have been tested in the flow regime $R e_{\mathrm{L}} \in\left[\begin{array}{ll}4.5 \times 10^{4} & 3.8 \times 10^{5}\end{array}\right]$.

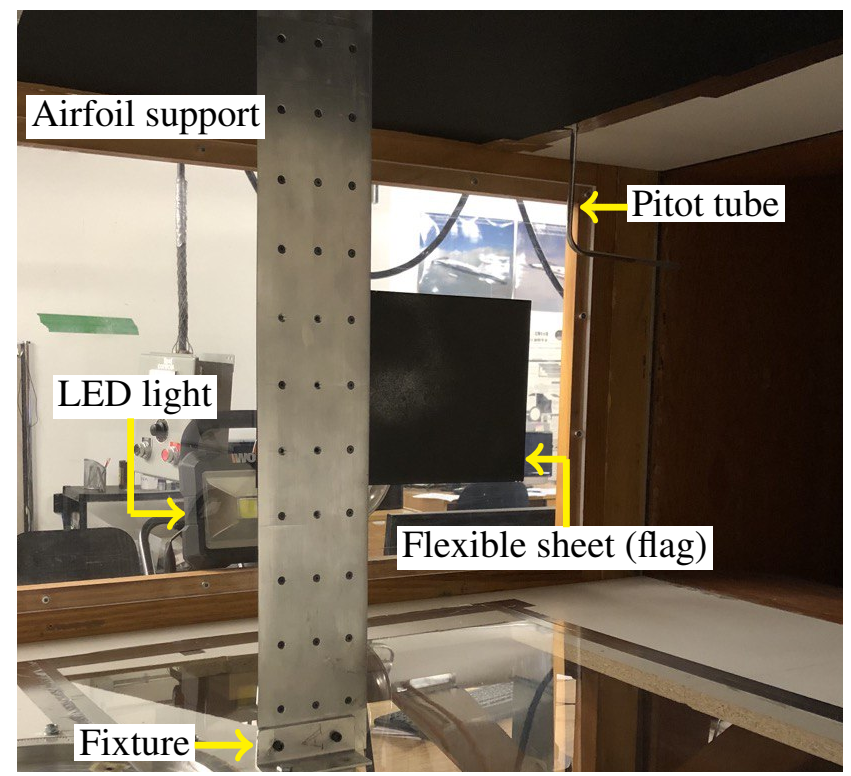

Figure 3: Experimental apparatus mounted in the test-section of the wind tunnel. Shown are the airfoil support secured by two sets of metallic fixtures against the top and bottom windows of the test-section, the flexible sheet (flag) being tested, one of LED lights illuminating the bottom edge of the flag, and the Pitot tube to measure flow velocity. Not shown are the high-speed camera placed underneath the test-section, a function generator triggering the camera, a data acquisition interface, and a power supply.

An airfoil-shaped flagpole was designed and fabricated for clamping the trailing end of the flexible plate and securing it in the test-section of the wind tunnel. The flagpole has a removable segment, so that the trailing-edge of the flag can be inserted and securely fixed. The chord length of the airfoil was $100 \mathrm{~mm}$ and the spanwise length $610 \mathrm{~mm}$. A Pitot tube detecting static and total pressure was used to measure the flow velocity in the test-section, and a thermocouple was used to measure the air flow temperature and to calculate its density. It should be noted that the flagpole length is comparable to the length of the flags tested in the experiments. Nonetheless, experiments with an even longer rigid splitter plate ( $\sim 10$ times of the flag length) attached to the trailing edge of the flag showed that vortex shedding from the trailing edge has a minimal effect on the global dynamics of the flags used in the present experiments (refer to Tavallaeinejad et al. 2020c). It is noted that such a relatively long streamlined flagpole profile was required to ensure that the flow disturbance due to the support is minimal and that the flag remains firmly clamped at high air speeds, up to $30 \mathrm{~m} / \mathrm{s}$.

\subsection{Flexible sheets}

A number of flags made of pliable sheets of various materials and dimensions were utilized. Specimens were cut from the sheets of various materials, using a shear-cut or a $\mathrm{CO}_{2}$ laser-cut machine. The mechanical properties of the materials used in the experiments are provided by the MatWeb material property data. ${ }^{3}$

${ }^{3}$ www.matweb.com 
However, the damped natural frequency of several specimens was measured before each test through free vibration tests, and the ratio $E /\left(1-v_{\mathrm{p}}^{2}\right)$ was then calculated, which was found to be in good agreement with that obtained from MatWeb.

\subsection{Motion capture and data extraction}

Measurements were made using a high-speed camera FLIR Grasshopper3 (IMX174) through the transparent Plexiglas window underneath the test-section. The high-speed camera has a global shutter, minimizing motion blur, and operates at $163 \mathrm{fps}$ at a maximum resolution of $1920 \times 1200$ pixels; the exposure time can vary between $5 \mu \mathrm{s}$ and $31.9 \mathrm{~s}$. This is sufficient to capture approximately 16 frames for a flapping cycle. A fixed focal length lens was mounted on the image sensor. Depending on the flag dimensions, the object distance to the camera was adjusted to provide the required field of view with the maximum possible resolution.

The camera system enables tracing multiple points on the bottom edge of the flag and determining the tip slope of the inverted flag during deformation. To facilitate this, three small white dots were painted on the tip of the bottom edge of the specimens with titanium white fluid, and were illuminated using LED lights. These three dots served as targets for image analysis.

A MATLAB image-processing script was developed for extracting the displacements of the three points from the video feeds. As shown in Figure 4(a), the leading-edge slope $\psi(L, t)$ at each frame was determined, using a linear fit to the three points. The choice of presenting the results in terms of $\psi(L, t)$ stems from the uniqueness of the angle of rotation as compared to the tip transverse deflection. More specifically, as shown in Figure 4(b), the maximum dimensionless transverse deflection, $w(L, t) / L$, over a cycle of oscillation hits the maximum value 0.86 , and remains almost constant with a slight variation. Thus, for such extremely large-amplitude oscillations, the motion cannot be fully described by looking at the transverse motion only. For the tip rotation angle, on the other hand, there exists a unique value, which can be used to reliably determine the state of the system.

Additionally, the farthest point from the clamped end was traced to determine the tip transverse and longitudinal deflections of the flag, $w(L, t)$ and $u(L, t)$, respectively, in each frame (see Figure 4$)$. The length of each flag at rest was used as the reference image for calibration.
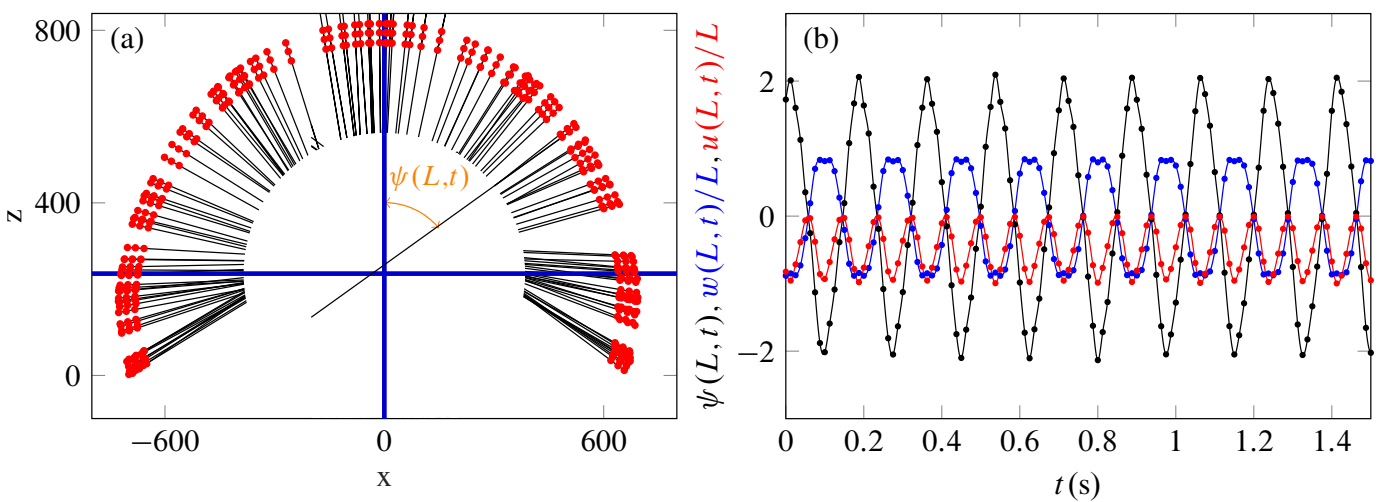

Figure 4: (a) Estimation of the tip angle rotation $\psi(L, t)$ using [-] a linear fit to [O] the three detected points in each frame; (b) [-] $\psi(L, t),[-] w(L, t)$ and $[-] u(L, t)$; the sampling rate is $80 \mathrm{~Hz}$.

The tip rotation was plotted against flow velocity to construct the bifurcation diagram and obtain information on the dynamic system behaviour. Using available displacements and tip-slope data and the corresponding velocities, phase portraits and Poincaré maps were created. Subsequently, the frequencies of oscillation were found from the Power Spectral Density (PSD) of each signal with the sampling frequency of $80 \mathrm{~Hz}$, using a rectangular window of the same length as that of the signal. For demonstration purposes and qualitative observations, the entire bottom edge of some specimens was painted white, and was traced in the image frames via a MATLAB script based on the edge detection technique. This allows constructing the time laps of flag position at different flow speeds and provides insight into the qualitative aspects of flag motion. It should be noted that the dynamics of the bottom edge is used in experiments to represent the flag flapping motion. Wider flags may exhibit considerable spanwise bending and torsional displacements which are not captured experimentally.

\section{General observations on the dynamics}

In this section, some of the general observations made from the present experiments with large- and very-low-aspect-ratio flags are described in detail. In particular, effects of the aspect ratio on the onset of instabilities (or regimes), their sequence and their associated features are discussed. 
Figure 5 depicts the dynamical states of an inverted flag obtained experimentally in different flow regimes. The first and second rows show, respectively, the states for inverted flags with $R=0.05$ and $R=1.0$,

(a)

(c)

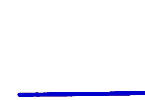

(d)

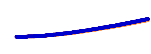

(e)

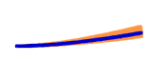

(b)

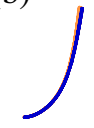

(g)

(h)

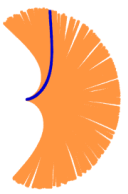

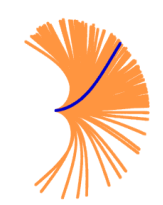

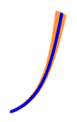

Figure 5: Experimental observations. Top row: time superimposed images of an inverted flag with $A=0.05$ at different regimes, (a) stretched-straight and (b) fully-deflected. Bottom row: superimposed images of an inverted flag with $R=1.0$ at different regimes, (c) stretched-straight, (d) buckled, (e) small-amplitude asymmetric flapping, (f) large-amplitude flapping, (g) aperiodic (chaotic) flapping, (h) and fully-deflected regimes.

which are, in fact, typical of those for very-low- and large-aspect-ratio inverted flags. Each subfigure has been obtained by superimposing the deformed shapes of the flag, corresponding to different instants of motion/time.

The first experimental results on the dynamics of inverted flags were presented in the seminal paper by Kim et al. (2013) who identified three consecutive regimes (or modes) of dynamical behaviour, namely the stretched-straight mode, the flapping mode and the fully deflected mode, as the flow velocity was increased. The shapes of these modes are similar to those shown in Figure 5(c), (f), and (h), respectively. Following Kim et al. (2013), measurements by Sader et al. $(2016 a, b)$ have revealed that the aspect ratio of the flag is a key parameter in the dynamics of inverted flags. They found a threshold, below which the dynamics of the inverted flag changes dramatically. More specifically, Sader et al. (2016b) showed that inverted flags with $R \lesssim 0.1$ do not undergo the same sequence of regimes as outlined above, but they transition directly from the undeflected mode to the fully deflected mode, that is from subfigure (a) to (b) in Figure 5, without encountering any intermediate regimes. Moreover, in addition to the three main dynamical regimes, Sader et al. (2016a) observed that the inverted flag exhibits a transition to chaos at high flow speeds, similarly to what is observed from Figure 5(g).

Soon after, Yu et al. (2017) conducted some experiments in a water channel and reported two additional dynamical regimes, namely the small-amplitude deflected (buckled) mode and the biased mode (smallamplitude asymmetric flapping around the buckled position), as shown respectively in Figs. 5 (d) and (e). Interestingly, these two regimes have also been identified in low-Re simulations by Goza et al. (2018) and Ryu et al. (2015) (both for $R e \lesssim 200$ ), as well as in high-Re simulations by Gurugubelli \& Jaiman (2019) $\left(R e=3 \times 10^{4}\right)$ and the analytical model proposed in Section 2.3; see also Tavallaeinejad et al. $(2020 b)$. Nevertheless, these regimes have not been observed in air-flow experiments yet.

\section{Dynamics of very-low-aspect-ratio inverted flag}

The bifurcation diagram in Figure 6(a) shows the stable equilibrium states for a very-low-aspect-ratio (or slender) inverted flag made of polycarbonate; more specifically, $R=0.05$ ( $L=160 \mathrm{~mm}$ and $H=8.0 \mathrm{~mm}$ ), $\mu=0.2\left(\rho_{\mathrm{p}}=1200 \mathrm{~kg} \mathrm{~m}^{-3}\right.$ and $\left.h=0.76 \mathrm{~mm}\right)$, and $\Pi / U=0.2366$; the measured tip rotation angles are plotted as a function of the flow speed. The results shown here are in a good qualitative agreement with those reported by Sader et al. (2016b). Specifically, the inverted flag is initially stable for relatively small flow velocities and remains at its original equilibrium position. At $U_{\mathrm{LAD}}^{+}=24.5 \mathrm{~m} / \mathrm{s}$, the flag jumps to a state with a highly curved shape; in that state, it undergoes some small-amplitude turbulence-induced vibrations. As the flow velocity is decreased, the flag does not return to equilibrium. Instead, it keeps performing low-amplitude vibrations about the deformed shape with decaying curvature. Eventually, at a much smaller flow velocity, $U_{\mathrm{LAD}}^{-} \lesssim 12.2 \mathrm{~m} / \mathrm{s}$, the flag jumps back to the original configuration, thus displaying a strongly subcritical pitchfork bifurcation. This indicates the existence of unstable equilibria in the $\left[U_{\mathrm{LAD}}^{+} U_{\mathrm{LAD}}^{-}\right]$range and determines a margin for coexisting attractors around the two stable equilibria. The presence of the unstable equilibria and the associated flag shapes were determined by Sader et al. (2016b) using an electric circuit, part of which was the flag-pole system.

Figure 6(b) shows the variation of the frequency of weak small-amplitude vibrations caused by perturbations in the flow, as a function of the flow speed. In this case, the highest peak in the FFT corresponds to the 
$\Pi$

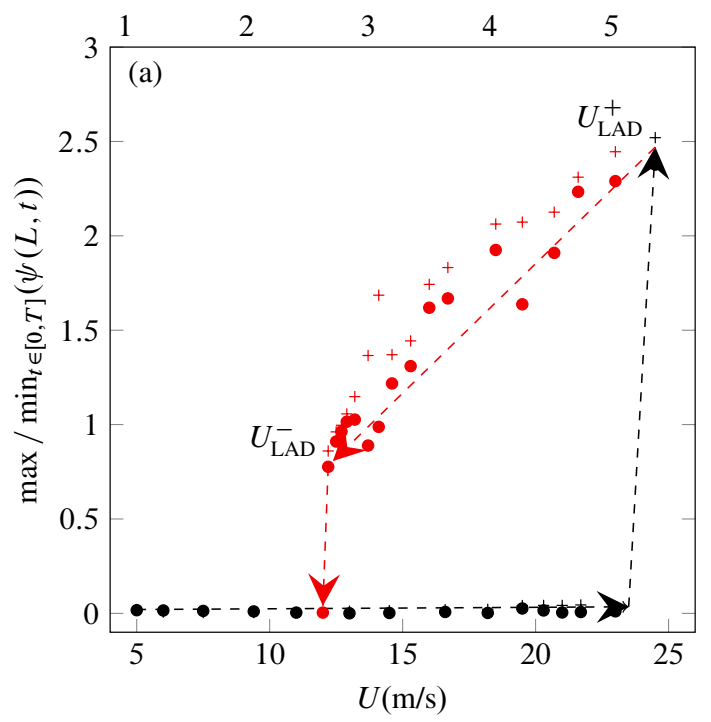

$\Pi$

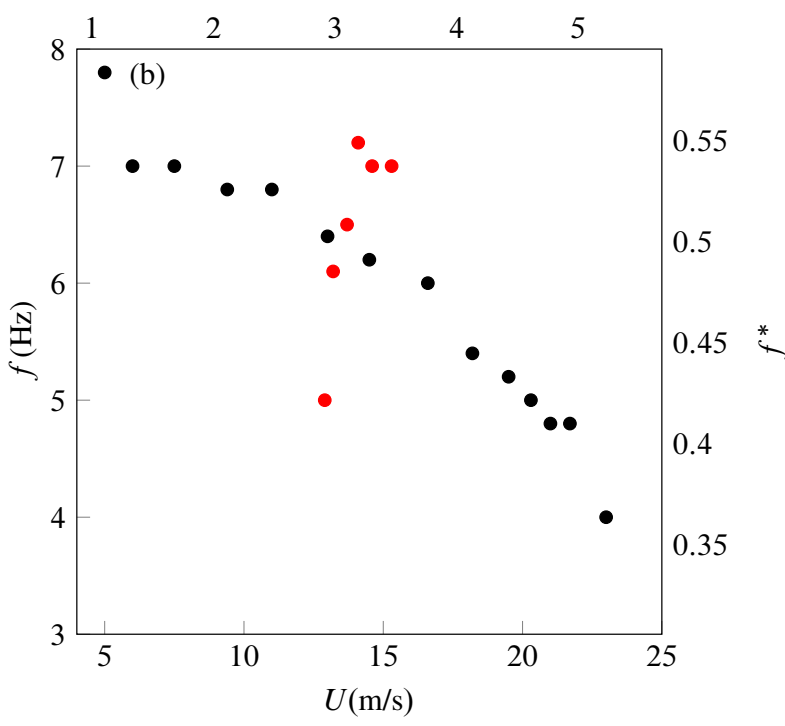

Figure 6: (a) Bifurcation diagram of a slender $(R=0.05)$ polycarbonate inverted flag with $L=160 \mathrm{~mm}$, $H=8.0 \mathrm{~mm}, h=0.76 \mathrm{~mm}$, showing $[+]$ the maximum and [0] the minimum amplitude of vibrations. (b) Frequency of weak small-amplitude vibrations around the static positions. Black and red symbols correspond to the wind-speed sweep up and down, respectively.

natural frequency of the flag-airflow system. As expected, the frequency of vibration around the undeflected equilibrium decreases as the flow velocity approaches to $U_{\text {LAD }}^{+}$where divergence (buckling) occurs. Similarly, the frequency of vibrations around the fully-bent state decreases as the flow velocity is swept down. It should be noted that no distinct frequency could be identified for vibrations around the fully-bent state and $U>16 \mathrm{~m} / \mathrm{s}$.

The results corresponding to the very-low-aspect-ratio inverted flag, such as the shapes shown in the first row of Figure 5, show a rather simple sequence of dynamical states (as compared to the large-aspect-ratio flags, discussed in Section 6.1), that is stretched-straight $\rightarrow$ fully deflected, which confirms the observations and measurements made by Sader et al. (2016b).

\section{Dynamics of large-aspect-ratio flags}

\subsection{Bifurcation diagram for the large-aspect-ratio flag}

Figure 7(a) shows the bifurcation diagram of Poincaré points for an inverted flag with $R=1$ ( $L=150 \mathrm{~mm}$ and $H=150 \mathrm{~mm}$ ), $\mu=0.2$ (cut from a polycarbonate sheet with $\rho_{\mathrm{p}}=1200 \mathrm{~kg} \mathrm{~m}^{-3}$ and $h=0.76 \mathrm{~mm}$ ), and $\Pi / U=0.1987$.

The tip rotation angle is shown as a function of the flow velocity. The trigger used for the Poincaré sections is that the tip velocity goes through zero (as in Goza et al. 2018). Hysteresis cycles are obtained by sweeping up and down the flow velocity. The notation $U^{+}$and $U^{-}$is used for critical flow velocities obtained via sweeping up and down, respectively. It can be seen that the flag remained straight at low flow velocities. However, as the flow velocity was increased, at $U_{\mathrm{D}}=7.2 \mathrm{~m} / \mathrm{s}$, the initially straight flag bent slightly to a stable deformed equilibrium. At $U_{\mathrm{SAF}}^{+}=8.2 \mathrm{~m} / \mathrm{s}$, the flag exhibited relatively small amplitude oscillations about the deformed shape. Following this motion, the flag entered the large-amplitude flapping regime at $U_{\mathrm{LAF}}^{+}=8.6 \mathrm{~m} / \mathrm{s}$, which eventually led to a chaotic regime with aperiodic motion at $U=13.4 \mathrm{~m} / \mathrm{s}$. Finally, at $U_{\text {LAD }}^{+}=14.1 \mathrm{~m} / \mathrm{s}$, the flag bent fully to one side, displaying small-amplitude oscillations about the deformed shape.

Once the flag entered the large-amplitude flapping regime, the flow velocity was decreased, and the flag returned to either of the straight/buckled/small-amplitude flapping regimes at $U_{\mathrm{LAF}}^{-}=8.1 \mathrm{~m} / \mathrm{s}$ in all three cases, indicating a subcritical onset of the flapping, resulting in a hysteresis loop, as also observed by Kim et al. (2013). Similarly, the fully-bent state exhibited hysteresis as the flow velocity was swept down; the flag returned to either the chaotic or large-amplitude regime at $U_{\mathrm{LAD}}^{-}=13.5 \mathrm{~m} / \mathrm{s}$.

The dominant frequency of oscillations as a function of the flow velocity is shown in Figure 7(b). The dominant frequency was found from the power spectral density (PSD) plots. Note that, even in the chaotic flapping regime, one dominant peak in the PSD was observed (see Figure 16).

The reduced-frequency (or Strouhal number), defined as $f_{\mathrm{R}}=f A_{\max } / U$ with $A_{\max }$ being the peak-to-peak amplitude, is plotted in Figure 7(c) versus the flow velocity, only for the range in which the inverted flag exhibits periodic flapping. Note that $A_{\max } \approx 0$ for $U<8.2$ and $U>14.1$ and thus $f_{\mathrm{R}} \approx 0$, and that is 

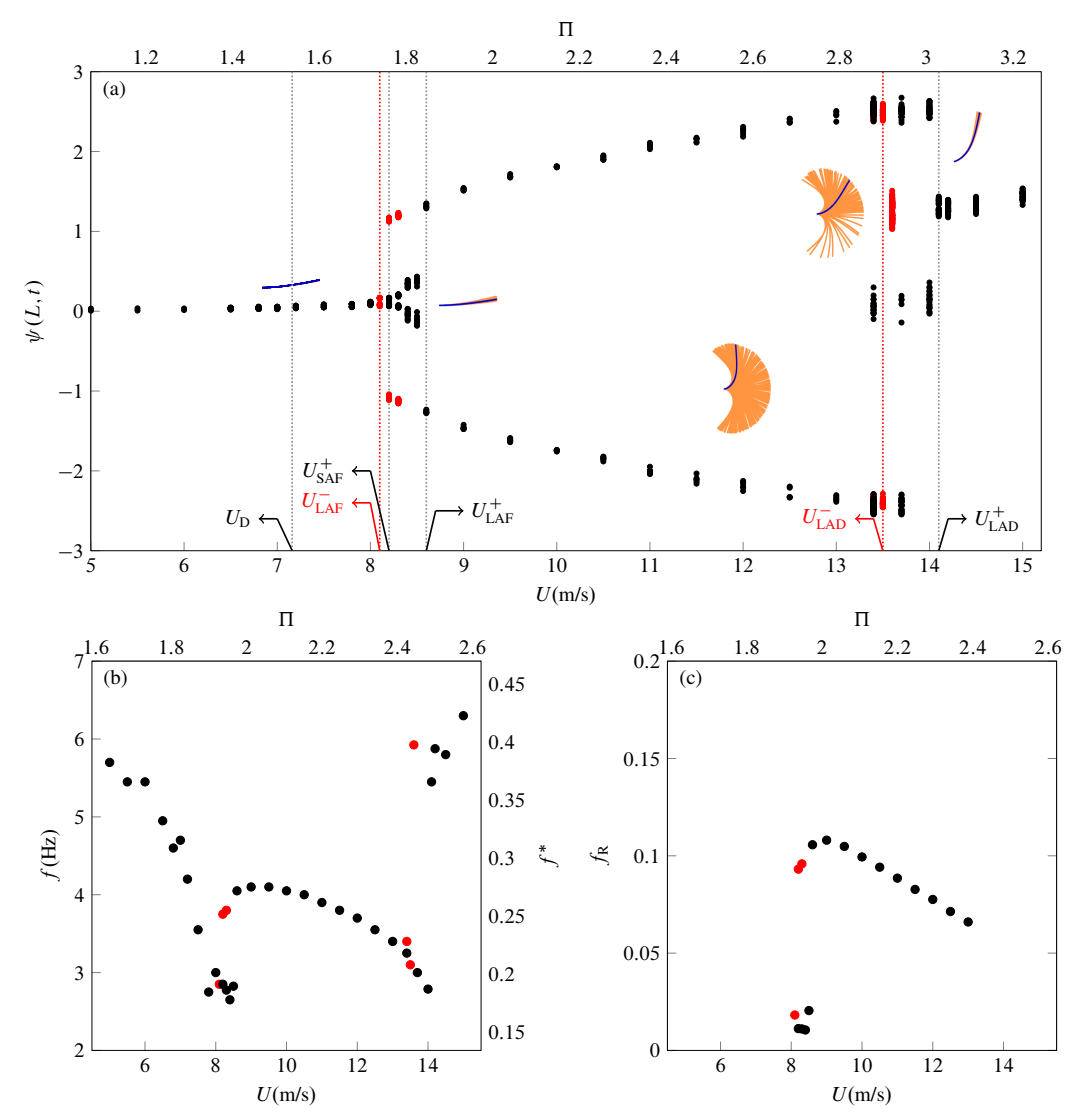

Figure 7: (a) Bifurcations of Poincaré sections for a polycarbonate inverted flag with $R=1$ ( $L=150 \mathrm{~mm}$, $H=150 \mathrm{~mm}, h=0.76 \mathrm{~mm}$ ). Black and red markers correspond to the flow velocity sweep up and down, respectively. $U_{\mathrm{D}}$, onset of small-amplitude static deformation; $U_{\mathrm{SAF}}$, onset of small-amplitude flapping; $U_{\mathrm{LAF}}$, onset of large-amplitude flapping; $U_{\mathrm{LAD}}$, onset of large-amplitude static deformation. Superscripts + and indicate critical flow velocities via sweeping up and down, respectively. (b) Flapping frequency at different flow velocities corresponding to the dominant peaks in the PSD plot. (c) Strouhal number $f_{\mathrm{R}}=f A_{\max } / U$ as a function of flow velocity.

why no points in those flow regimes are shown in Figure 7(c). In the small-amplitude flapping regime, $A_{\max }$ reaches a maximum value of $\sim 62 \mathrm{~mm}$, while in the large-amplitude flapping regime, $A_{\max }$ can get as high as $\sim 270 \mathrm{~mm}$. Hence, the reduced frequencies in the small-amplitude regime are significantly smaller than those associated with the large-amplitude flapping regime; $\max \left(f_{\mathrm{R}}\right)=0.11$ occurs around $U=9 \mathrm{~m} / \mathrm{s}$.

From the bifurcation diagram in Figure 7(a) and the results shown in the second row of Figure 5, the sequence of dynamical states for a large-aspect-ratio flag is organised as follows: stretched-straight $\rightarrow$ buckled $\rightarrow$ asymmetric deformed-flapping $\rightarrow$ large-amplitude flapping $\rightarrow$ chaotic $\rightarrow$ fully deflected, which is consistent with observations made by Yu et al. (2017) in a water channel (note, however, that the chaotic regime was not observed in that study). It should be noted that the asymmetric deformed-flapping regime (see Figure 5(e)) was not observed in some test cases, and the flag transitioned immediately form the undeflected or buckled state to large-amplitude flapping; this is discussed in more detail in Section 6.7.

In the following subsections, the characteristics of the system in each regime are discussed in more detail.

\subsection{Pre- and post-divergence regimes}

While the existence of a divergence instability is expected from the measurements of Kim et al. (2013), as well as the results obtained via the theoretical model outlined in Section 2.3 and other theoretical studies in the literature (e.g., Sader et al. 2016a, Gurugubelli \& Jaiman 2015, Goza et al. 2018), it was not always possible to determine the onset of divergence accurately. In fact, many flags used in both earlier and present experiments possessed an intrinsic initial curvature along their length due to geometric imperfections, mainly in the form of the first mode shape of a cantilevered beam, leading to non-zero initial tip deflections. Moreover, if the flag was sufficiently wide and thin, it sagged slightly under its own weight. Increasing the flow velocity in such cases caused a growth in the deviation from the perfectly straight shape, giving rise to a gradual, rather than an abrupt, development of divergence.

In order to pinpoint the onset of divergence, the method described by (Païdoussis, 2016, Chapter 8) and Païdoussis et al. (2002) is employed. As discussed the following paragraphs, due to inflow perturbations, the flag never remains completely static, vibrating with a very small amplitude around the deformed 
shape. Hence, the minimum/maximum dimensionless transverse displacement at the flag leading edge, i.e. $\min / \max _{t \in[0, T]}(w(L, t))$, is plotted as a function of the flow velocity, as shown in Figure 8 . As seen, a

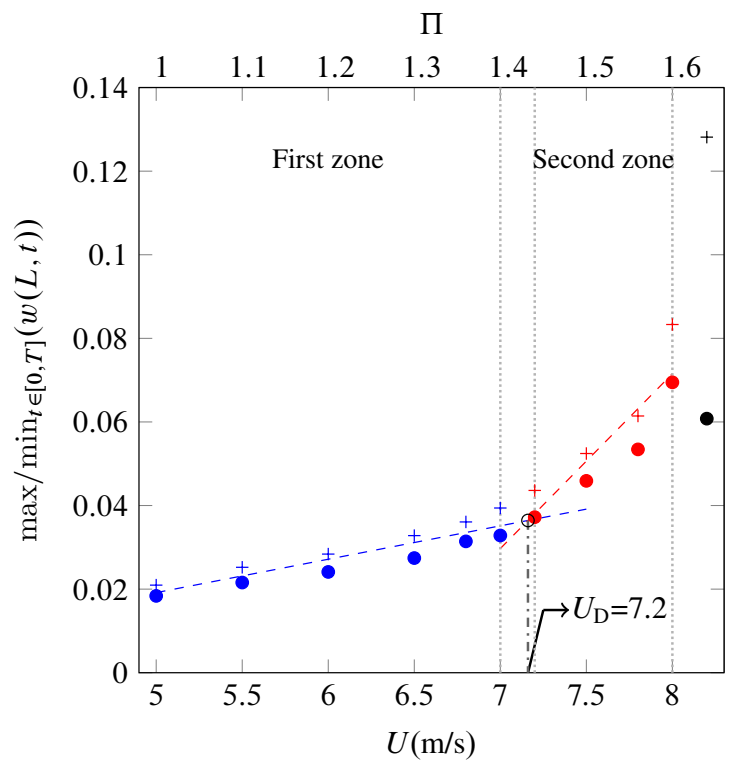

Figure 8: Dimensionless transverse displacement of the leading edge or free-end of the flag in Figure 7 as a function of the flow velocity; $[+]$ maximum and $[0]$ minimum amplitude of vibrations; dashed lines are the linear fits to the average values of displacement. The marked difference between the slopes of the lines indicates the emergence of divergence.

gradual increase of bending (or deformation) has replaced the ideal precipitous emergence of the divergence instability. Two zones may be identified in the figure: (i) the first zone, $U<7.0 \mathrm{~m} / \mathrm{s}$, wherein non-zero amplitudes of deflection increase moderately due to growth of initial geometric imperfections of the flag; (ii) the second zone, $7.3 \mathrm{~m} / \mathrm{s}<U<8.0 \mathrm{~m} / \mathrm{s}$ approximately, wherein the increase in $w(L, t)$ with $U$ is much sharper, signaling the emergence of divergence. The average transverse displacements in each zone are then fitted via two straight lines, the intersection of which determines the critical flow velocity for divergence, i.e. $U_{\mathrm{D}}=7.2 \mathrm{~m} / \mathrm{s}$ in this case.

It is recalled that the minimum attainable flow speed in the test-section of the wind tunnel was around $5 \mathrm{~m} / \mathrm{s}$. Consequently, in some cases, for flags of large aspect ratio, divergence occurred at a flow velocity very close to, or even below the minimum speed. In such cases, the onset of divergence is pinpointed roughly, taking into account the deflection of the flag at zero flow velocity. In addition, it should be noted that the thresholds used for defining the two zones, are specific to experiments and need some degree of experience and subjective judgement.

Figures 9 and 10 show time history, PSD, phase-plane portrait, and probability density function (PDF) ${ }^{4}$ plots for vibrations in the pre-buckling $(U=5.5 \mathrm{~m} / \mathrm{s})$ and post-buckling $(U=8.0 \mathrm{~m} / \mathrm{s})$ zones, respectively.

Bursts of aperiodic motions can be observed in the time history plot in Figure 9(a). In addition, the phase-plane portrait looks erratic and dense. The PDF plot presents an almost convex region around $w(L, t) \sim$ $0.023-0.024$, but is still double-masted, indicating the mostly visited locations by the flag tip during the motion. Moreover, the PSD plot shown in Figure 9(b) is typical of a turbulence-induced vibration spectrum. The few peaks seen in the PSD plot are broad-banded and of low power. The highest peak in the spectrum at $f=5.85 \mathrm{~Hz}$ corresponds to the natural frequency of the flag-airflow system. A superharmonic of this frequency, $4 f=23.61 \mathrm{~Hz}$, can also be seen in the frequency spectrum. The source of the other two peaks could not be identified. All the plots in Figure 9 suggest a very-small-amplitude random-like motion about a deflected shape.

By increasing the flow velocity, very small-amplitude non-coherent vibrations, induced by turbulence/unsteadiness in the flow, become more damped. Moreover, the dominant frequency decreases.

Interestingly, the dominant frequency of vibrations for $U=8.0 \mathrm{~m} / \mathrm{s}$, which lies in the post-buckling zone, has shifted further towards zero, though it does not become zero (see Figure 10(b)). This observation suggests

\footnotetext{
${ }^{4}$ In order to avoid making assumptions about the distribution of a signal, a kernel distribution is employed to estimate PDF. For any real values of $x$, the kernel density estimator formula is given by $\hat{f}_{h}(x)=\frac{1}{m h} \sum_{j=1}^{m} K\left(\frac{x-x_{j}}{h}\right)$, where $K$ denotes the kernel smoothing function, $h$ is a smoothing parameter, and $x_{j}$ are random samples drawn from an unknown distribution of density $f$. It is recalled that the PDF of a period-1 signal presents two peaks at the extremes of the displacement, where motion is slow, and hence the likelihood of finding the system there is high; Gaussian-like distribution is associated with random or chaotic motion (refer to Païdoussis 2016).
} 


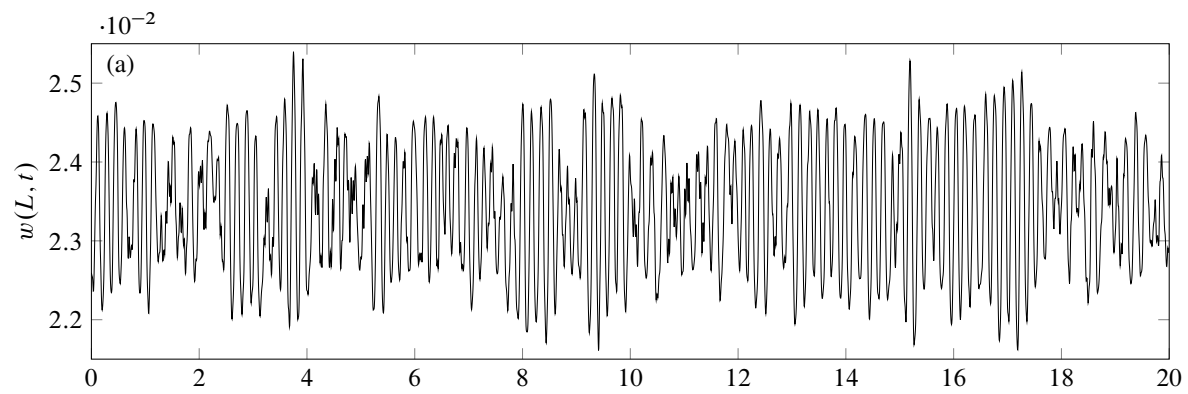

$t(\mathrm{~s})$
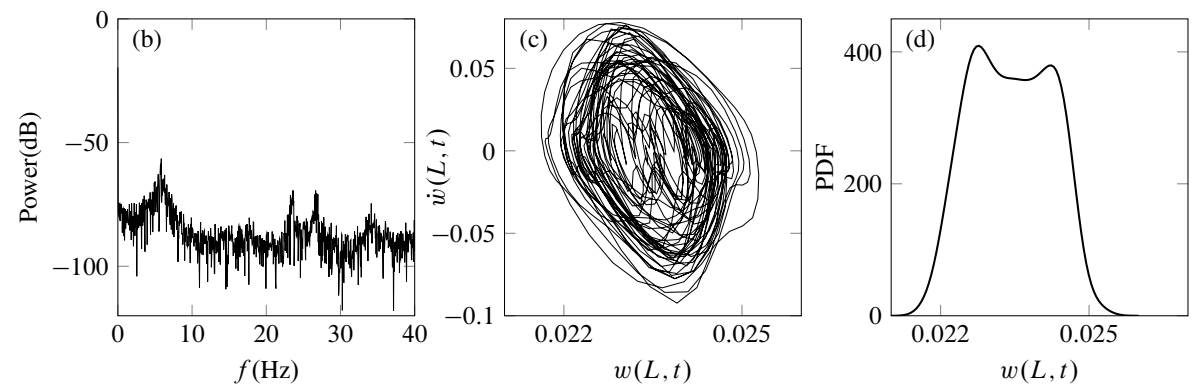

Figure 9: Experimental results at $U=5.5 \mathrm{~m} / \mathrm{s}(\Pi=1.09)$ for the flag of Figure 7 with a random-like motion around the deflected equilibrium: (a) time history, (b) PSD, (c) phase-plane portrait, and (d) PDF of the free-end displacement of the flag.

the existence of a non-vanishing stiffness in the system in the buckled state, in contrast to what would be expected from a linear dynamics analysis, but perfectly reasonable from a nonlinear dynamics perspective.

\subsection{Small-amplitude asymmetric flapping regime}

As opposed to divergence instability, the onset of oscillatory instabilities, such as small-amplitude asymmetric flapping, $U_{\mathrm{SAF}}^{+}$, and large-amplitude flapping, $U_{\mathrm{LAF}}^{+}$, could usually be easily identified through spotting the transition from random-like vibrations to coherent periodic oscillations. However, similarly to the deflected regime, the small-amplitude flapping around the buckled shape was not observed in all the cases tested. Nevertheless, in some cases this regime extended over a considerable range of the flow velocity.

As discussed in Section 6.2, the PSD plots for $U<U_{\mathrm{SAF}}^{+}$show that the response of the inverted flag to flow turbulence is broad-banded; on the other hand, as $U_{\mathrm{SAF}}^{+}$is approached, one frequency becomes dominant as shown in Figure 11.

Moreover, two prominent peaks in the PDF plot at the extremities of the transverse displacement, and an asymmetric limit cycle that develops after $U_{\mathrm{SAF}}^{+}$are seen. This regime extends over the range $8.2 \leq U \leq 8.5$, with the amplitude of flapping increasing with flow velocity.

The frequency content of the displacement at different flow velocities is plotted in the form of a spectrogram in Figure 12. The colors show the distribution of power $(\mathrm{dB})$ for the different frequencies in the signal. As seen, one can easily confirm that the system does not exhibit a broad-banded response. Instead, a concentration of power/energy is observed around $f \simeq 3 \mathrm{~Hz}$, establishing that this is the dominant frequency. At higher flow velocities within the small-amplitude flapping regime, apart from the dominant frequency at $f \simeq 3 \mathrm{~Hz}$, only an additional peak in PSD plots emerges, which corresponds to a superharmonic of the dominant frequency.

The underlying mechanism for small-amplitude asymmetric flapping is not vortex-induced vibrations (VIV). In this flow regime, oscillations are of low reduced-frequencies, much smaller than the typical values for VIV (i.e., $f_{\mathrm{R}} \approx 0.1-0.15$ ); see Figure $7(\mathrm{c})$. Goza et al. (2018) also reported that a non-VIV mechanism is responsible for the establishment of this regime for a wide range of mass ratios. The type of instability involved was shown by Goza et al. to emerge via a Hopf bifurcation, also predicted by the theoretical model of Section 2.3.

\subsection{Large-amplitude flapping regime}

Large-amplitude flapping occurs at $U_{\mathrm{LAF}}^{+}=8.6 \mathrm{~m} / \mathrm{s}$. While the amplitude of flapping increases with the flow velocity, its dominant frequency decreases (refer to Figure 7). The time-history, PSD, phase-plane and PDF plots for the motion at $U=8.6 \mathrm{~m} / \mathrm{s}$ are shown in Figure 13. The single-loop phase-plane portrait, the double-masted PDF, and the existence of a dominant frequency (i.e. $f_{1} \sim 4.43 \mathrm{~Hz}$ ) in the PSD plot (along with a weak superharmonic at $3 f_{1}$ ), all indicate a periodic (period-1) motion.

At sufficiently high flow velocities, about $U=13.0 \mathrm{~m} / \mathrm{s}$, the leading-edge transverse displacement displays a double-humped shape at the peak (see Figure 14), indicating that the tip slope has passed $\pi / 2$ 

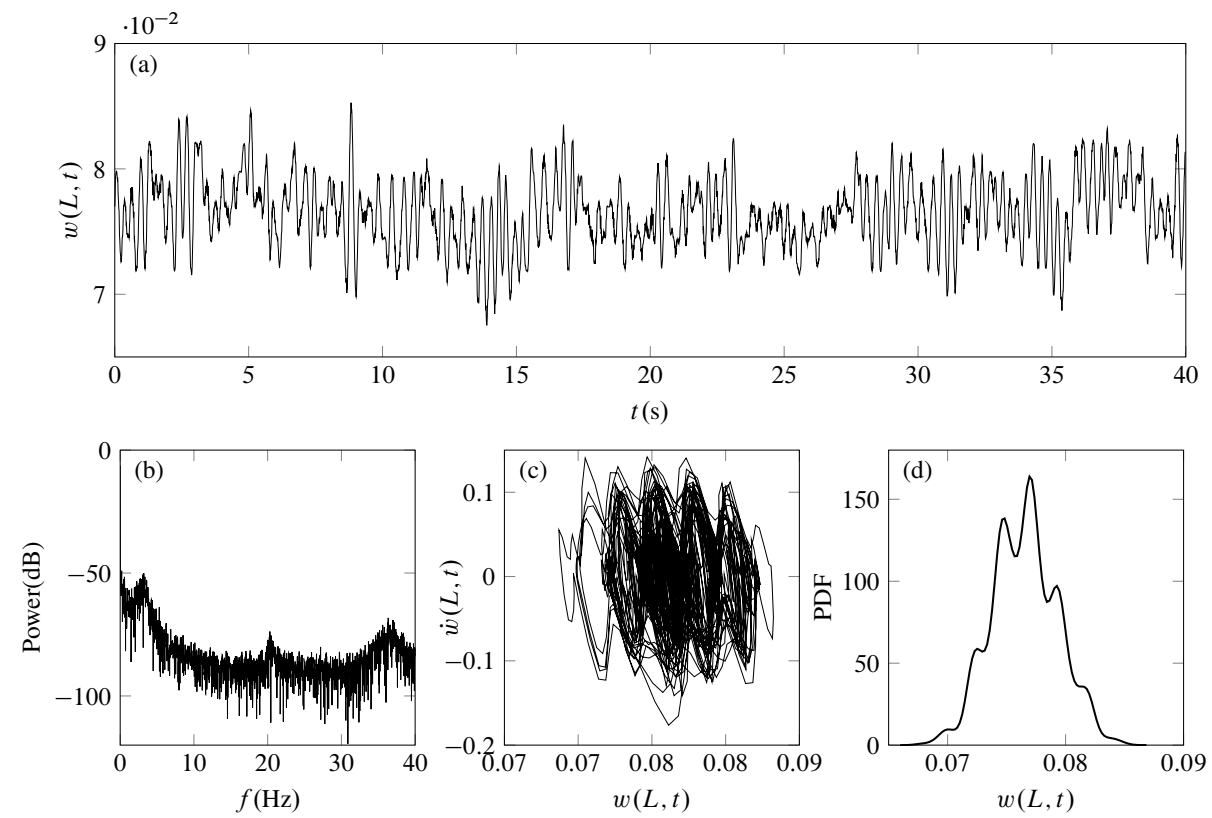

Figure 10: Experimental results at $U=8.0 \mathrm{~m} / \mathrm{s}(\Pi=1.59)$ for the flag of Figure 7 with a random motion around the deflected equilibrium; (a) the time history, (b) PSD, (c) phase-plane portrait, and (d) PDF of the response.

with the free-end pointing downstream. This results in a double-loop phase-plane plot, suggesting a period-2 motion (as shown in the inset of Figure 14(c)). However, the phase-plane plot for the tip rotation angle shown in Figure 14(d) forms a single loop, confirming a periodic motion with one dominant frequency. Nonlinear effects, notably associated with such extremely large deflections of the flag, are likely responsible for the evolution of multi-harmonics in the PSD plot.

Once the flow velocity was decreased below the point where the large-amplitude flapping first appeared, the symmetric oscillation persisted with reduced amplitude, until the flag returned to small-amplitude oscillations at $U_{\mathrm{LAF}}^{-}=8.1 \mathrm{~m} / \mathrm{s}$ (see Figure 7(a)); this is another attribute of the nonlinear behaviour of the flag. More specifically, there is a considerable hysteresis in the threshold of large-amplitude flapping with increasing and decreasing flow, suggesting the existence of two saddle-node bifurcations in the system response.

The spectrogram for the large-amplitude flapping regime is shown in Figure 15. The plot shows that, as the flow velocity is increased, the single-dominant-frequency (accompanied with some superharmonics) spectrum, indicating a periodic motion, evolves into a broad-banded spectrum (see the lower right-hand corner at $U=13.4 \mathrm{~m} / \mathrm{s}$ ). This gradual increase in the dynamic activity and the increase in the low-frequency content suggest the possibility of chaotic motion at higher flow velocities, as discussed in the next section.

\subsection{Chaotic regime}

The first study in which the aperiodic regime, immediately following large-amplitude flapping but at sufficiently high flow velocity, prior to the fully deflected state, was identified as involving chaotic motion is attributed to Sader et al. (2016a). They compared extracts of the motion time histories with nearly identical initial trajectories and showed that such time series deviate rapidly from one another in a finite time, and hence established the existence of chaos.

Although sensitivity to changes in initial conditions is one of the key characteristics of chaotic motion, which is often determined by the Lyapunov exponent, here we employ other standard measures for detecting chaotic oscillations, in the narrow range of $13.4 \lesssim U \lesssim 14.0$.

The characteristics of the dynamics at $U=13.4 \mathrm{~m} / \mathrm{s}$ are shown in Figure 16, specifically the time-history, PSD, phase-plane portrait, PDF, and autocorrelation plots. The time trace of the motion shows that the oscillations take place mostly around the undeflected equilibrium, although they are sometimes biased towards the deflected equilibrium. The phase-plane plot reflects the existence of an overriding, rather strong periodic component in the motion. The PSD plot is quite broad-banded, yet a dominant frequency can still be noticed. The autocorrelation decays with time quite fast, showing loss of memory after a few cycles of motion. On the other hand, the PDF does not have a Gaussian distribution, still possessing fairly sharp peaks at the extremities of the motion with a suspension-bridge-like shape. Taking all the measures into account, one may conclude that a "limited or narrow-banded" chaotic motion exists at $U=13.4 \mathrm{~m} / \mathrm{s}$.

As the flow velocity is increased, the chaotic motion becomes stronger. As seen, for example, from Figure 17, a 'two-well potential' form appears in the phase-plane portrait, and the PDF plot has filled up between the two peaks at the limits of the motion (i.e., $w(L, t) \simeq \pm 0.75$ ). The strength of the chaotic motion 

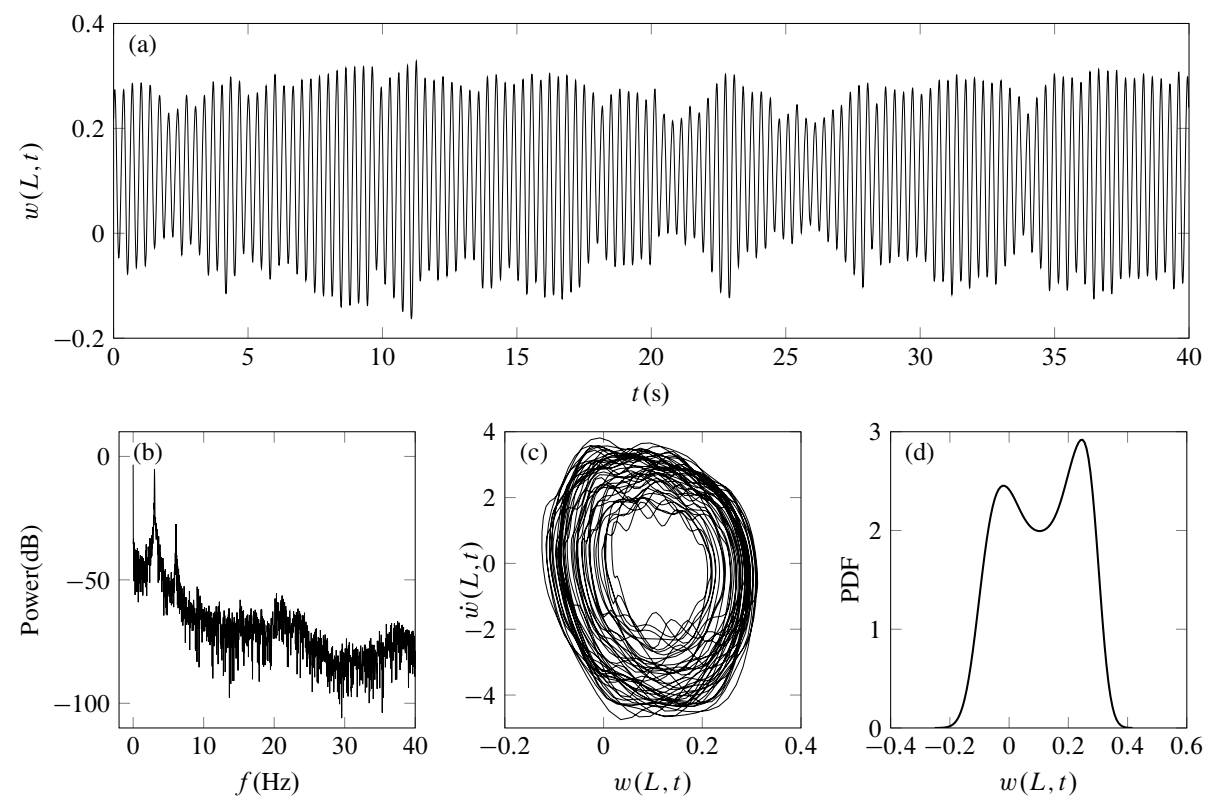

Figure 11: Experimental results at $U=8.5 \mathrm{~m} / \mathrm{s}(\Pi=1.69)$ for the flag of Figure 7 with a periodic motion around the deflected equilibrium; (a) time history, (b) PSD, (c) phase-plane portrait, and (d) PDF of the response.

is also evident from the time series, by examining the relative duration of the intermittent quasi-random behaviour (compare Figure 17(a) with Figure 16(a)).

\subsection{Fully-deflected regime}

At a sufficiently high flow velocity, $U_{\mathrm{LAD}}^{+}=14.1 \mathrm{~m} / \mathrm{s}$, the inverted flag eventually stops flapping and displays a highly-deflected curved shape. In this flow regime, the flag bends almost fully to one side, so that the upstream portion of the flag is subjected to cross-flow rather than axial flow. Thus, the flag resembles a bluff body subjected to a cross-flow, with periodic vortex shedding from both the leading- and trailing-edges. Similarly to the large-amplitude flapping regime, hysteresis was observed in the transition from the flapping to the fully-deflected regime.

The dynamical characteristics of the flag at $U=14.5 \mathrm{~m} / \mathrm{s}$ are shown in Figure 18, where the leadingedge slope, $\psi(L, t)$, is used instead of the transverse displacement. Random-like fluctuations about a large mean-value in the time-history, a single-masted PDF about a large non-zero angle, a quite erratic phase-plane portrait, and a broad-banded PSD plot, all lend some support to the existence of random-like vibrations, due to flow perturbations about the highly-deflected body. It is worth mentioning that the dominant frequency of oscillation increases with $U$ (refer to Figure 7 (b)).

\subsection{A note on the sequence of observed dynamical states or regimes}

Before closing this section, it ought to be mentioned that there is a discrepancy between the present experimental results and the measurements made by Kim et al. (2013) insofar as some dynamical states are considered. More specifically, in the experimental results shown in Figure 7, the existence of the buckled state and small-amplitude flapping around the buckled position was demonstrated. Although these observations are consistent with the measurements of Yu et al. (2017) in a water channel and the experiments by Murugesan Pazhani \& Acharya (2019) with serrated flags, they differ from the experimental observations of Kim et al. (2013) in airflow, who reported a spontaneous jump from the stretched-straight state to large-amplitude flapping. Nevertheless, the same regime transitions shown in Figure 7 have been found computationally by Goza et al. (2018) and analytically by the model of Section 2.3. In addition, the existence of the (unstable) deflected equilibrium was revealed experimentally by Sader $e t a l$. (2016b) by introducing additional damping. In another experimental study, Fan et al. (2019) reported that "small-scale flutter" was observed at flow velocities below the large-amplitude flapping threshold. This, however, might be due to flow turbulence, which was not small in their experiments.

The first conceivable explanation for the discrepancy among experimentally-observed sequences of dynamical states could be the presence of an initial curvature due to imperfections along the flags. As shown by Cossé et al. (2014), increasing the initial angle of attack leads to a gradual growth in the amplitude of oscillation. It was also noted that flags with larger initial curvature are more prone to exhibit deformed-flapping motion. However, in many tests performed by the authors, in which the deflected and small-amplitude asymmetric flapping regimes were observed, the initial tip deflection was $\Delta / L \leq 0.02$. The existence of such 


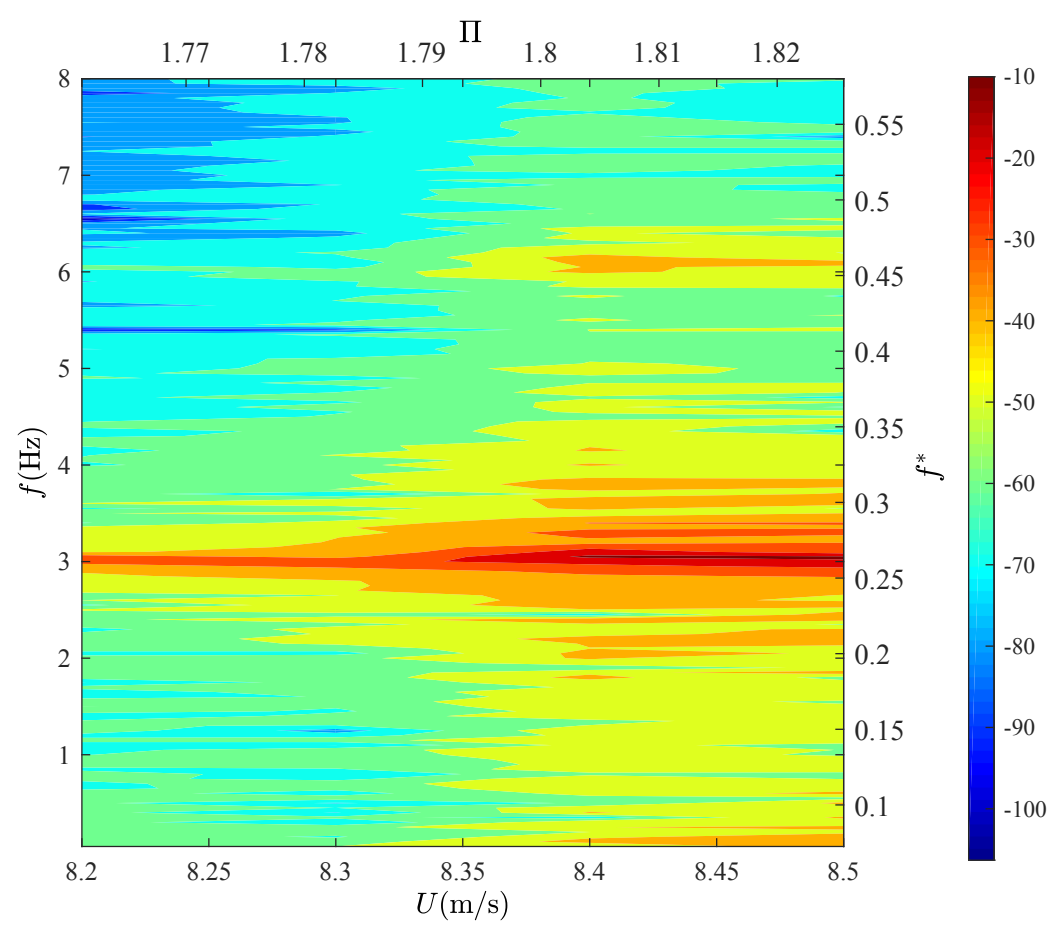

Figure 12: Experimental spectrograms for the flag of Figure 7 with respect to flow speed; magnitude in dB.

a small imperfection and its consequences may not wholly explain the difference in the post-critical behaviour, as discussed above.

Another plausible reason for the above-mentioned discrepancy could be the existence of a bistable region wherein large-amplitude flapping occurs. This phenomenon would give rise to the possibility of a jump of the flag from static undeflected equilibrium to large-amplitude flapping motion directly, without passing through other states. Vortex-shedding, overshoots during flow speed increments, or incoming flow turbulence may provide sufficiently strong disturbances for the jump to occur. This can also be interpreted in the context of the nonlinear model of Section 2.3, where the possibility of multiple solutions reflects an initial-conditiondependent behaviour. Interestingly, it was recognized that for most cases, $U_{\mathrm{LAF}}^{-}<U_{\mathrm{SAF}}^{+}<U_{\mathrm{LAF}}^{+}$. Clamping imperfections and the pre-stress introduced during material fabrication and in the mounting procedure might also lead to the suppression of small-amplitude vibrations, and a direct transition to the large-amplitude flapping regime.

\subsection{The effects of material on the dynamics}

The seminal experimental study by Kim et al. (2013) with inverted flags with $\mu=0.38$ (in air) and $\mu=320$ (in water) revealed that, unlike for conventional flags, the mass ratio does not significantly change the flow velocity at which flapping occurs, while other dynamical characteristics, such as the frequency and amplitude of oscillations, are noticeably affected. However, they reported that the flow velocity at which flapping ceases does depend on $\mu$ (it varies by a factor of 2 from air to water).

In another experimental study, Fan et al. (2019) reported slight differences in the flow velocities at which flapping occurs/ceases for inverted flags with $\mu$ between $\mu=0.61$ and $\mu=0.40$. Interestingly, the value of the dimensionless bending stiffness (dimensionless flow velocity) for which flapping ceases for $\mu=0.61$ is systematically larger (smaller) than that for $\mu=0.4$; see (Fan et al., 2019, Figure 4(a)).

The purpose of the experiments described in this section was to obtain some data for flags with low values of mass ratio (heavy flags), below the range explored in previous experimental studies. The control parameters in the experiments (in air) in order to vary the mass ratio parameter, $\mu=\rho_{\mathrm{f}} L /\left(\rho_{\mathrm{p}} h\right)$, are $L, h$, and $\rho_{\mathrm{p}}$. Increasing $L / h$ decreases the natural frequency of the structure, leading to a reduction in the dimensional flow velocity at which the large-amplitude flapping, initiated by a divergence instability, occurs. Note that the reduction in the flow velocity with $L / h$ is faster than with increasing $\mu$ (see Eq. (3)). Thus, covering a wide range of values for $\mu$ via only changing the length-to-thickness ratio was not attainable due to the minimum flow velocity wind-tunnel limitation, i.e. $\sim 5 \mathrm{~m} / \mathrm{s}$. As a result, it was decided to utilize flags made of different materials (different material density $\rho_{\mathrm{p}}$ ), though it is recognized that this way the structural damping of the flags would vary also. For that reason, the differences in behaviour, which are qualitative as well as quantitative, can more precisely be attributed to differences in material more than mass ratio. For all specimens, the width $H$ is adjusted such that the aspect ratio remains constant at $R=1.2$. The specimens used in these experiments are listed in Table 1. 

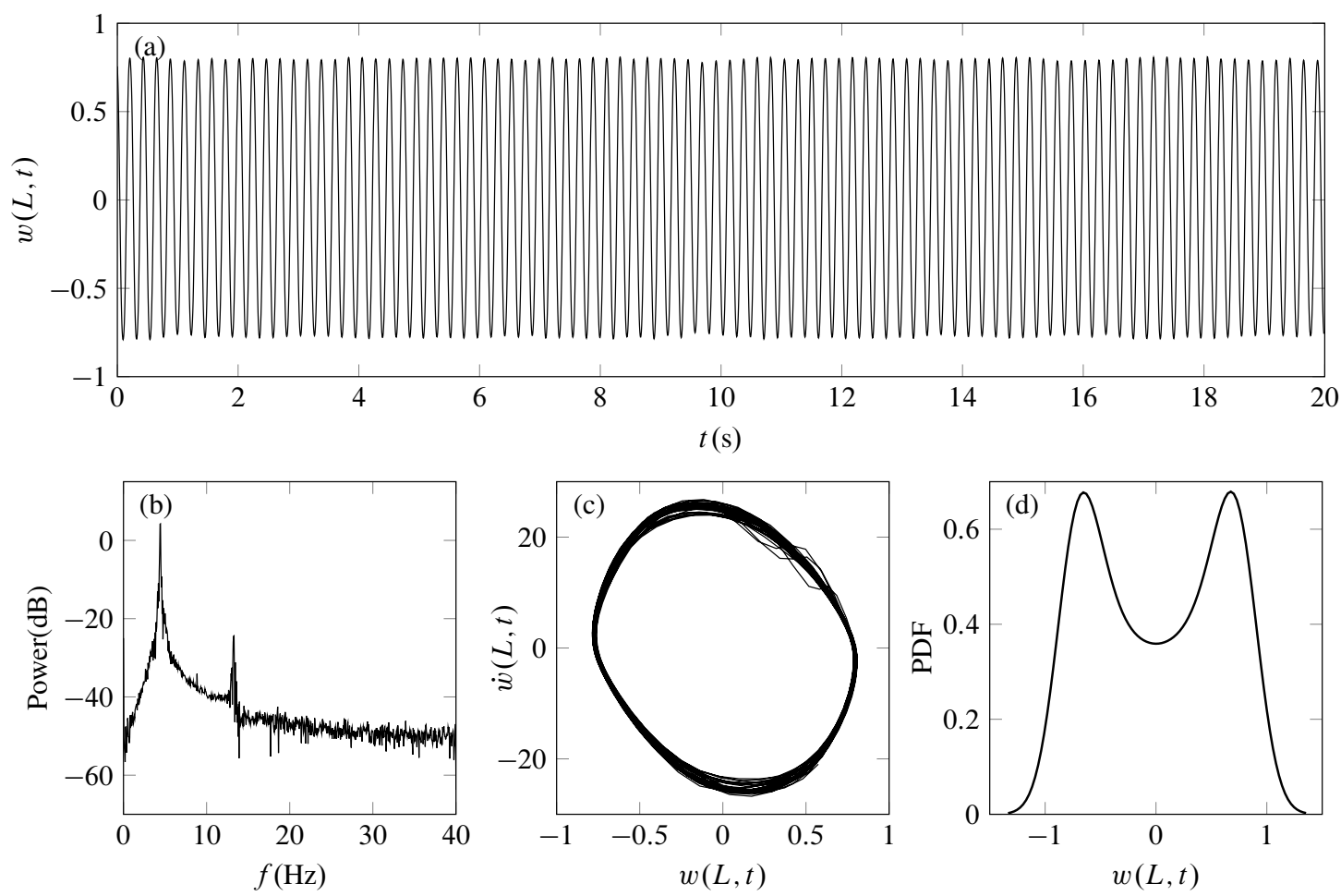

Figure 13: Experimental results at $U=8.6 \mathrm{~m} / \mathrm{s}(\Pi=1.71)$ for the flag of Figure 7 with a periodic motion around the undeflected equilibrium; (a) time history, (b) PSD, (c) phase-plane portrait, and (d) PDF of the response.

\begin{tabular}{|c|c|c|c|c|c|c|c|c|}
\hline Test & Material & $\begin{array}{c}\text { Material density } \\
\rho_{\mathrm{p}}\left(\mathrm{kg} \mathrm{m}^{-3}\right)\end{array}$ & $\begin{array}{c}\text { Young's modulus } \\
E(\mathrm{GPa})\end{array}$ & $\begin{array}{c}\text { Poisson's ratio } \\
v_{\mathrm{p}}\end{array}$ & $\begin{array}{l}\text { Length } \\
L(\mathrm{~mm})\end{array}$ & $\begin{array}{l}\text { Height } \\
H(\mathrm{~mm})\end{array}$ & $\begin{array}{c}\text { Thickness } \\
h(\mathrm{~mm})\end{array}$ & $\begin{array}{l}\text { Mass ratio } \\
\rho_{\mathrm{f}} L /\left(\rho_{\mathrm{p}} h\right)\end{array}$ \\
\hline 1 & 260 Brass & 8430 & 100 & 0.32 & 127.0 & 152.0 & 0.25 & 0.07 \\
\hline 2 & Stainless steel & 7600 & 193 & 0.29 & 70.0 & 84.0 & 0.08 & 0.15 \\
\hline 3 & Zinc & 7135 & 97 & 0.25 & 240.0 & 288.0 & 0.20 & 0.20 \\
\hline 4 & Polycarbonate & 1200 & 2.4 & 0.38 & 190.0 & 228.0 & 0.76 & 0.25 \\
\hline 5 & Polypropylene & 946 & 1.5 & 0.38 & 200.0 & 240.0 & 0.76 & 0.33 \\
\hline
\end{tabular}

Table 1: Physical dimensions and properties of the flags made of different materials and different mass ratios but the same aspect ratio $R=1.2$.

Experimental results on the effects of the mass ratio on the dynamics of the flag are presented in Figure 19 in the form of bifurcation diagrams. As seen, all the flags underwent large-amplitude flapping motion, except the zinc flag (Test \#3). More specifically, although the inverted flag made of zinc was of sufficiently high aspect ratio for flapping to occur $(R \gtrsim 0.1)$, it performed but a few cycles of oscillation at $\Pi \approx 1.52$ before buckling abruptly with a large-amplitude steady deflection (see Figure 19(c)). This was a surprising occurrence. It should be noted that the zinc flag had an intrinsic curvature in the vertical direction, which is known to increase the stiffness of the system via $E h \kappa^{2}$ ( $\kappa$ being the curvature) and in turn, raise the instability threshold (see experiments on conventional flags by Eloy et al. 2012).

As seen from Figure 19(e), the polypropylene flag (Test \# 5), which is the lightest flag in the group, performed large-amplitude asymmetric (on one side only) flapping as soon as the wind tunnel was turned on, i.e. $\Pi=0.38 U=1.90$. That is why there are no data points prior to $\Pi=1.90$. By increasing the flow velocity, the flapping became symmetric and the flag crossed the $\psi=0$ line, until flapping ceased at $\Pi=2.85$. As the flow velocity was reduced from $\Pi=2.85$, the flag became deflected towards one side and remained in that state until the minimum flow velocity. The flag eventually returned to the initial position at an unmeasured flow velocity once the wind tunnel was turned off.

As seen from Figure 19(a), the inverted flag made of brass (Test \#1) undergoes the regular sequence of regimes as discussed in Section 4 , but the large-amplitude flapping motion became asymmetric at $\Pi \approx 2.20$, and then stopped abruptly at $\Pi \approx 2.26$. This may be explained as being due to local yielding of the material at the root of the flag. This led to a permanent deformation, such that the flapping then occurred around the new flag configuration.

Figure 19(b) shows the bifurcation diagram for the stainless steel inverted flag, displaying a considerable amount of hysteresis between the onset (wind-speed sweep up) and cessation (wind-speed sweep down) of large-amplitude flapping. It is of interest to record that the inverted flag was able to perform second-mode 

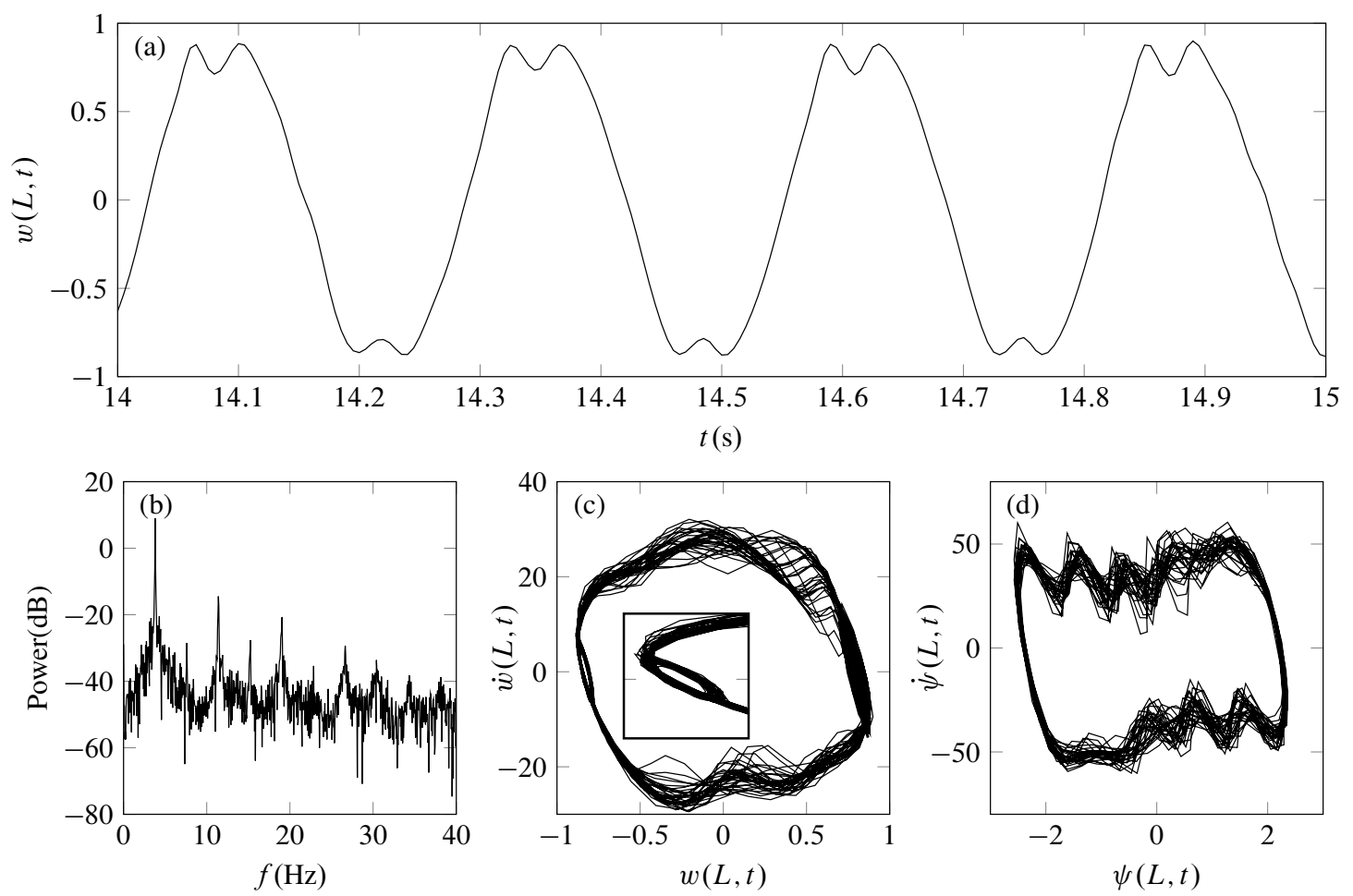

Figure 14: Experimental results at $U=13.0 \mathrm{~m} / \mathrm{s}(\Pi=2.58)$ for the flag of Figure 7 with a periodic motion around the undeflected equilibrium; (a) time history of the response, (b) PSD, (c) phase-plane portrait of the transverse displacement, and (d) phase-plane portrait of the tip rotation angle.

flutter at very high flow velocities, at $\Pi=6.24$, with dominant frequency $f=27.3 \mathrm{~Hz}$ and with the free-end pointing downstream (not shown in the bifurcation diagram). It should be noted that the flag returned to its original rest configuration, once the wind tunnel was turned off, with no visual evidence of permanent deformation.

\section{Effects of aspect ratio on the dynamics}

The first set of experiments which shed light on the effects of the geometry on the dynamics of the flag were conducted by Cossé et al. (2014). Their measurements with flags of aspect ratios in the range of $0.08<A<2.08$ showed that the aspect ratio of a flag can dramatically alter the dynamics. They reported that while inverted flags with sufficiently large aspect ratios may undergo a flapping motion prior to a fully deflected state, very-low-aspect-ratio inverted flags transition directly from the stretched-straight to the fully deflected state without entering the flapping regime. This work was pursued by Sader et al. $(2016 a, b)$ who conducted experiments on inverted flags over a wide range of aspect ratios. In the first study, they reported that the critical flow velocity for the onset of large-amplitude flapping decreases as the aspect ratio is increased, while the critical value at which the flag enters the fully-deflected regime is unaffected, leading to a broader range of flapping for larger aspect ratios. In the second paper, Sader et al. (2016b) examined the stability of very-low-aspect-ratio flags (down to $R=0.033$ ) and found a wide range of bistability for slender inverted flags, wherein the flag may exhibit either the undeflected or fully deflected shape. Moreover, they reported a threshold for the aspect ratio $(R \lesssim 0.1)$ below which flapping did not occur.

In this section, the work of Sader et al. $(2016 a, b)$ is extended to examine the effects of aspect ratio on the global dynamical behaviour in inverted flags. In particular, we study the variations of the sequence of instabilities and the critical flow velocities for each regime, as well as the frequencies and amplitude of flapping as $R$ is varied.

Our preliminary experiments had shown the tendency of inverted flags with $R \gtrsim 0.1$ to exhibit largeamplitude flapping, prior to the development of the steady fully deflected shape, which agrees well with the measurements by Sader et al. (2016b). On the other hand, most flags with $A<0.1$ did not exhibit symmetric large-amplitude flapping; instead, they buckled abruptly. Therefore, it was decided to investigate the effects of the flag aspect ratio over each range, separately.

In the first set of experiments, polycarbonate plates with $h=0.76 \mathrm{~mm}$ and $h=1.02 \mathrm{~mm}$ and a constant length of $L=150 \mathrm{~mm}$ (hence, constant mass ratio $\mu=0.20$ and $\mu=0.15$, respectively) and different widths were fabricated. They cover a wide range of aspect ratios: $0.1 \leq R \leq 4$. The physical dimensions of the flags with $\mu=0.20$ used in the experiments are given in Table 2 . 


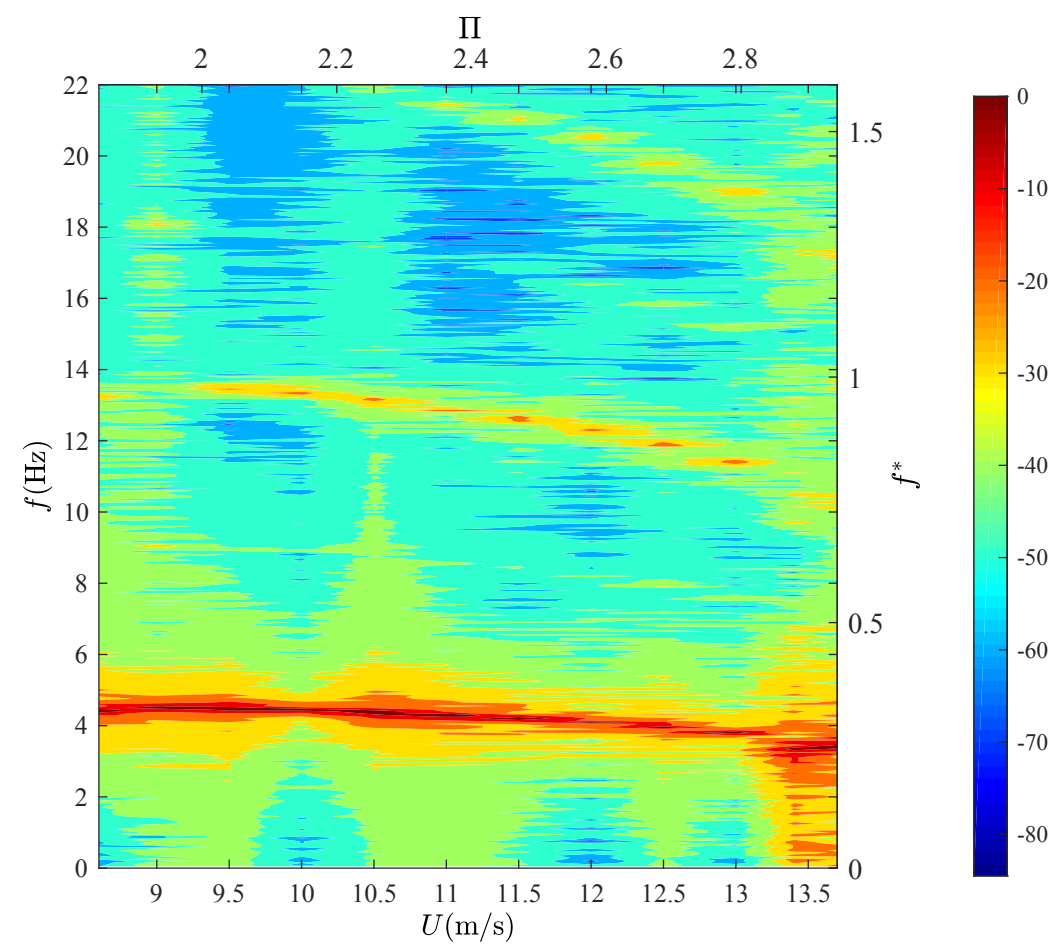

Figure 15: Experimental spectrogram for the large-amplitude flapping regime in Figure 7; power spectrum in dB.

\begin{tabular}{cccc|cccc}
\hline Test & $\begin{array}{c}\text { Length } \\
L(\mathrm{~mm})\end{array}$ & $\begin{array}{c}\text { Height } \\
H(\mathrm{~mm})\end{array}$ & $\begin{array}{c}\text { Aspect ratio } \\
R=H / L\end{array}$ & Test & $\begin{array}{c}\text { Length } \\
L(\mathrm{~mm})\end{array}$ & $\begin{array}{c}\text { Height } \\
H(\mathrm{~mm})\end{array}$ & $\begin{array}{c}\text { Aspect ratio } \\
R=H / L\end{array}$ \\
\hline L1 & 150.0 & 15.0 & 0.10 & L6 & 150.0 & 187.5 & 1.25 \\
L2 & 150.0 & 37.5 & 0.25 & L7 & 150.0 & 262.5 & 1.75 \\
L3 & 150.0 & 75.0 & 0.50 & L8 & 150.0 & 300.0 & 2.00 \\
L4 & 150.0 & 112.5 & 0.75 & L9 & 150.0 & 450.0 & 3.00 \\
L5 & 150.0 & 150.0 & 1.00 & L10 & 150.0 & 600.0 & 4.00 \\
\hline
\end{tabular}

Table 2: Physical dimensions of large-aspect-ratio polycarbonate flags used in the experiments; $E=2.4 \mathrm{GPa}$, $\rho_{\mathrm{p}}=1200 \mathrm{~kg} \mathrm{~m}^{-3}, v_{\mathrm{p}}=0.38, h=0.76 \mathrm{~mm}$ and $\mu=0.20$.

To further investigate the effects of $R$ on the stability of very slender inverted flags, another set of experiments was conducted using two polycarbonate flags of $H=8.0 \mathrm{~mm}$ and $H=16.0 \mathrm{~mm}$ with $h=$ $0.76 \mathrm{~mm}$ and initial length of $L=230 \mathrm{~mm}$. The flag was shortened after each test, which enabled covering the range of $0.035<R<0.1$; see Table 3 for the physical dimensions of the flags.

\begin{tabular}{cccc|cccc}
\hline Test & $\begin{array}{c}\text { Length } \\
L(\mathrm{~mm})\end{array}$ & $\begin{array}{c}\text { Height } \\
H(\mathrm{~mm})\end{array}$ & $\begin{array}{c}\text { Aspect ratio } \\
R=H / L\end{array}$ & Test & $\begin{array}{c}\text { Length } \\
L(\mathrm{~mm})\end{array}$ & $\begin{array}{c}\text { Height } \\
H(\mathrm{~mm})\end{array}$ & $\begin{array}{c}\text { Aspect ratio } \\
R=H / L\end{array}$ \\
\hline S1 & 230.0 & 8.0 & 0.035 & S5 & 230.0 & 16.0 & 0.070 \\
S2 & 200.0 & 8.0 & 0.040 & S6 & 200.0 & 16.0 & 0.080 \\
S3 & 175.0 & 8.0 & 0.046 & S7 & 175.0 & 16.0 & 0.090 \\
S4 & 160.0 & 8.0 & 0.050 & S8 & 160.0 & 16.0 & 0.100 \\
\hline
\end{tabular}

Table 3: Physical dimensions of very-low-aspect-ratio polycarbonate flags used in the experiments; $E=2.4 \mathrm{GPa}$, $\rho_{\mathrm{p}}=1200 \mathrm{~kg} \mathrm{~m}^{-3}, v_{\mathrm{p}}=0.38$, and $h=0.76 \mathrm{~mm}$.

The experimental results are summarized in the form of stability maps in Figure 20, highlighting the sensitivity of the foregoing bifurcations (i.e., $\Pi_{\mathrm{D}}, \Pi_{\mathrm{SAF}}^{+}, \Pi_{\mathrm{LAF}}^{+}, \Pi_{\mathrm{LAF}}^{-}, \Pi_{\mathrm{LAD}}^{+}$, and $\Pi_{\mathrm{LAD}}^{-}$; refer to Section 6.1 and Figure 7 for definitions) to the aspect ratio.

It is seen in Figure 20(a) that the critical value for divergence, $\Pi_{\mathrm{D}}$, decreases with increasing $R$. Similar trends can be seen for $\Pi_{\mathrm{SAF}}^{+}, \Pi_{\mathrm{LAF}}^{+}$, and $\Pi_{\mathrm{LAF}}^{-}$. Interestingly, these critical flow velocities are most strongly affected by the aspect ratio in the range $A \in[0.11 .0]$, while they become almost insensitive to $R$ for higher values of the aspect ratio. This observation suggests that the effects of the sides of the flag become less significant for $R \gtrsim 2$, implying that the flow may reasonably be considered two-dimensional over most length of the flag span.

On the other hand, the critical flow velocities for the onset of fully-deflected state $\left(\Pi_{\mathrm{LAD}}^{+}\right.$and $\Pi_{\mathrm{LAD}}^{-}$for increasing and decreasing flow speed, respectively) remain almost constant for the range of $R$ tested. This 

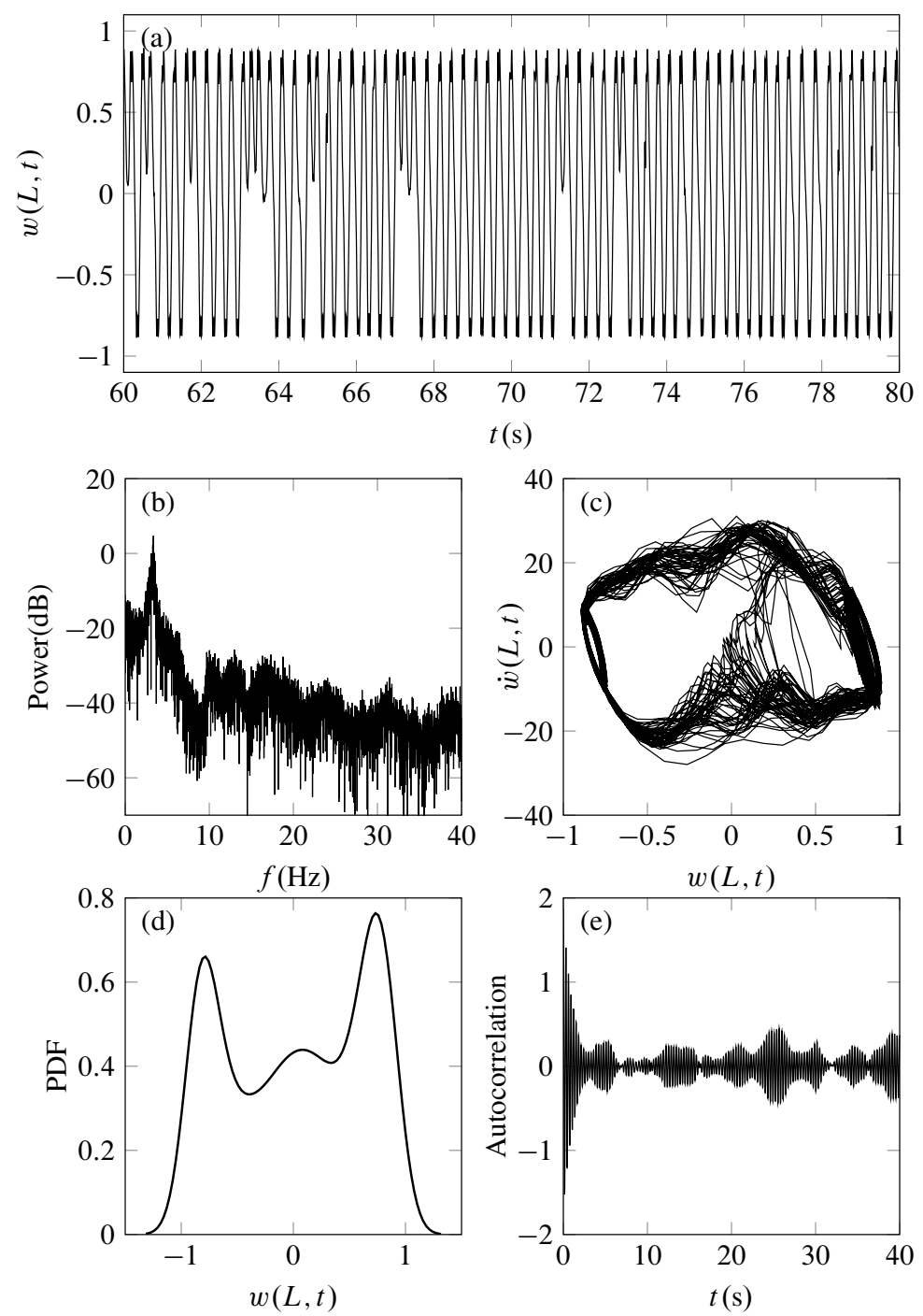

Figure 16: Experimental results at $U=13.4 \mathrm{~m} / \mathrm{s}(\Pi=2.66)$ for the flag of Figure 7 with an aperiodic (chaotic) motion; (a) time history of the response, (b) PSD, (c) phase-plane portrait, (d) PDF, and (e) autocorrelation.

means that as $R$ is increased, the flag performs large-amplitude flapping over a wider range of flow velocity. The insensitivity of $\Pi_{\mathrm{LAD}}^{+}$and $\Pi_{\mathrm{LAD}}^{-}$to $R$ has also been reported previously; for example, see Cossé et al. (2014). Interestingly, it can be seen in Figure 20 (a) that $\Pi_{\mathrm{LAD}} / \Pi_{\mathrm{LAF}} \approx 2$, in agreement with observations made by Fan et al. (2019) for inverted flags of trapezoidal shape.

Figure 20(b) shows similar results for $\mu=0.15$. In this case, no data points for $\Pi_{\mathrm{LAD}}^{+}$and $\Pi_{\mathrm{LAD}}^{-}$are provided, because the flags were destroyed at very high flow velocities prior to the development of the fully-deflected regime (probably due to the high-frequency large-amplitude flapping). Nevertheless, similar qualitative trends to those shown in Figure 20(a) can be seen. The sharp decrease in the onset of instabilities becomes smoother for $R \gtrsim 0.5$, signalling that two-dimensional flow characteristics become dominant.

Figure 21 shows the dimensionless flapping frequency $f^{*}$ (see Eq. (3) for the definition ${ }^{5}$ ) and amplitude for inverted flags with different aspect ratios. It should be noted that only the frequency of large-amplitude flapping is plotted in this figure; frequencies associated with small-amplitude and chaotic motions are excluded. It is seen that the flapping frequency increases with $R$, albeit moderately. It is also evident that for all cases, the increase in the flow velocity causes the frequency of oscillation to decrease until the motion becomes chaotic. Interestingly, the amplitude of oscillation also increases with $R$. Considering the dimensionless flow velocity $\Pi=1.9$, for example, the amplitude of flapping for the inverted flag with $R=0.5$ is substantially smaller than that for $R=4.0$; also, while for the former the tip rotation is less than $\pi / 2$, the latter flaps with the free-end pointing downstream. This may be attributed to an enhanced leading-edge vortex formation with increasing $R$. It is hypothesized that as $R$ is increased, the flow three-dimensionality due to side edges is diminished, which in turn allows for stronger and faster leading-edge vortex formation and shedding over a

\footnotetext{
${ }^{5}$ The reduced frequency is defined as $f_{\mathrm{R}}=f^{*} \sqrt{\mu} / \Pi$.
} 

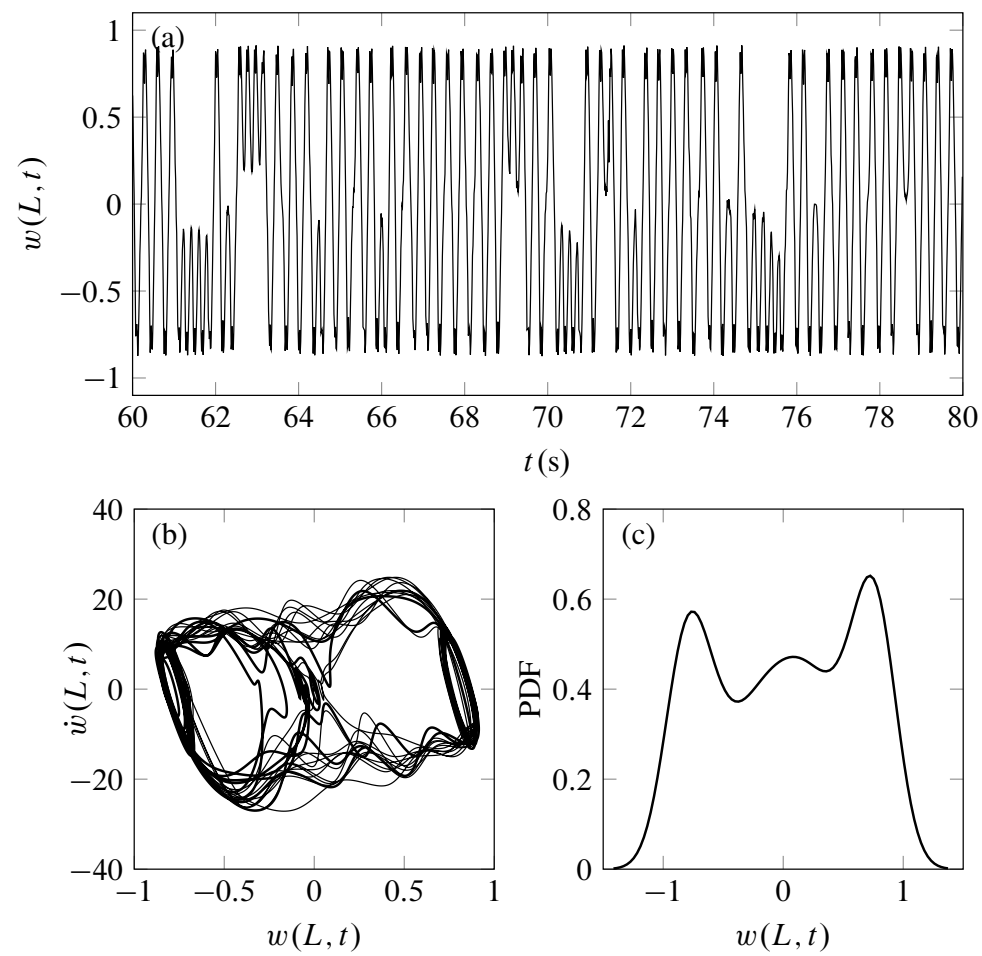

Figure 17: Experimental results at $U=13.7 \mathrm{~m} / \mathrm{s}(\Pi=2.72)$ for the flag of Figure 7 with an aperiodic (chaotic) motion; (a) time history of the response, (b) phase-plane portrait, and (c) PDF.

wider range of span.

Figure 22 illustrates how the dimensionless critical flow velocity for divergence, $\Pi_{D}$, is pinpointed for different values of the aspect ratio. As seen, divergence does not develop abruptly for large aspect ratios. It is recalled that divergence is very sensitive to imperfections (e.g., initial curvature along the length of the flag), and hence, its onset is difficult to pinpoint. Moreover, due to limitations of the wind tunnel insofar as the lowest operable wind speed is concerned, very large aspect ratio flags (i.e. $R=3.0$ and $R=4.0$ with $\mu=0.2)$ deflected at relatively low flow velocities $(U<5.0 \mathrm{~m} / \mathrm{s}$ ), effectively entering the second zone (i.e. buckled state) immediately, posing a challenge to pinpointing the divergence instability. Another point should also be mentioned here. Although the existence of a static divergence was implied in the results of Kim et al. (2013), to the best of the authors' knowledge, no experimental results have been reported, in that study or any other study in the literature, on the variation of the onset of divergence with the aspect ratio.

Figure 23 shows the stability boundary obtained for very-low-aspect-ratio (or slender) inverted flags. It should be mentioned that the critical value for $\Pi_{\mathrm{LAD}}^{+}$for slender flags can be viewed as the onset of divergence. In other words, the flow velocity at which a slender flag makes an unassisted jump to the large-amplitude deflected state indicates the onset of divergence via a subcritical pitchfork bifurcation (i.e. $\Pi_{\mathrm{P}}$ ). Similarly, $\Pi_{\mathrm{LAD}}^{-}$is equivalent to $\Pi_{S}$, where the saddle-node bifurcation materializes.

According to the experimental observations made by Sader et al. (2016b) and the theoretical predictions by Tavallaeinejad et al. (2020a), one may expect that $\Pi_{\mathrm{LAD}}^{+}$and $\Pi_{\mathrm{LAD}}^{-}$decrease as $R$ is increased. However, as seen in Figure 23, these critical values do not decrease monotonically with $R$; instead they vary within a narrow range. This may partially be justified by the fact that longer flags (and thus flags of lower aspect ratios) suffer more from inevitable initial imperfections (mainly curvature), displaying non-zero angles of attack, and hence leading to reduced values of $\Pi_{\mathrm{LAD}}^{+} \equiv \Pi_{\mathrm{p}}$ (see Section 2.2). It should be remarked that all slender flags tested in the experiment possessed a small tip deflection $0.01 \leq \Delta / L \leq 0.04$.

Another reason for the above might be that, as opposed to measurements obtained by Sader et al. (2016b), in the present experiments, the two jumps between the undeflected and fully deflected states occurred naturally (without any assistance/disturbance), both with increasing and decreasing flow velocity. Recalling that the response of slender inverted flags exhibits a wide range of bistability, and any perturbations arising from vortex-shedding, overshoots during flow velocity increments, or incoming flow turbulence may facilitate the jumps. Therefore, the measured values for $\Pi_{\mathrm{LAD}}^{+}\left(\Pi_{\mathrm{LAD}}^{-}\right)$would be higher (lower) if the initial position of the flags were adjusted and their motion were suppressed using an external pole, similarly to what was done by Sader et al. (2016b). 

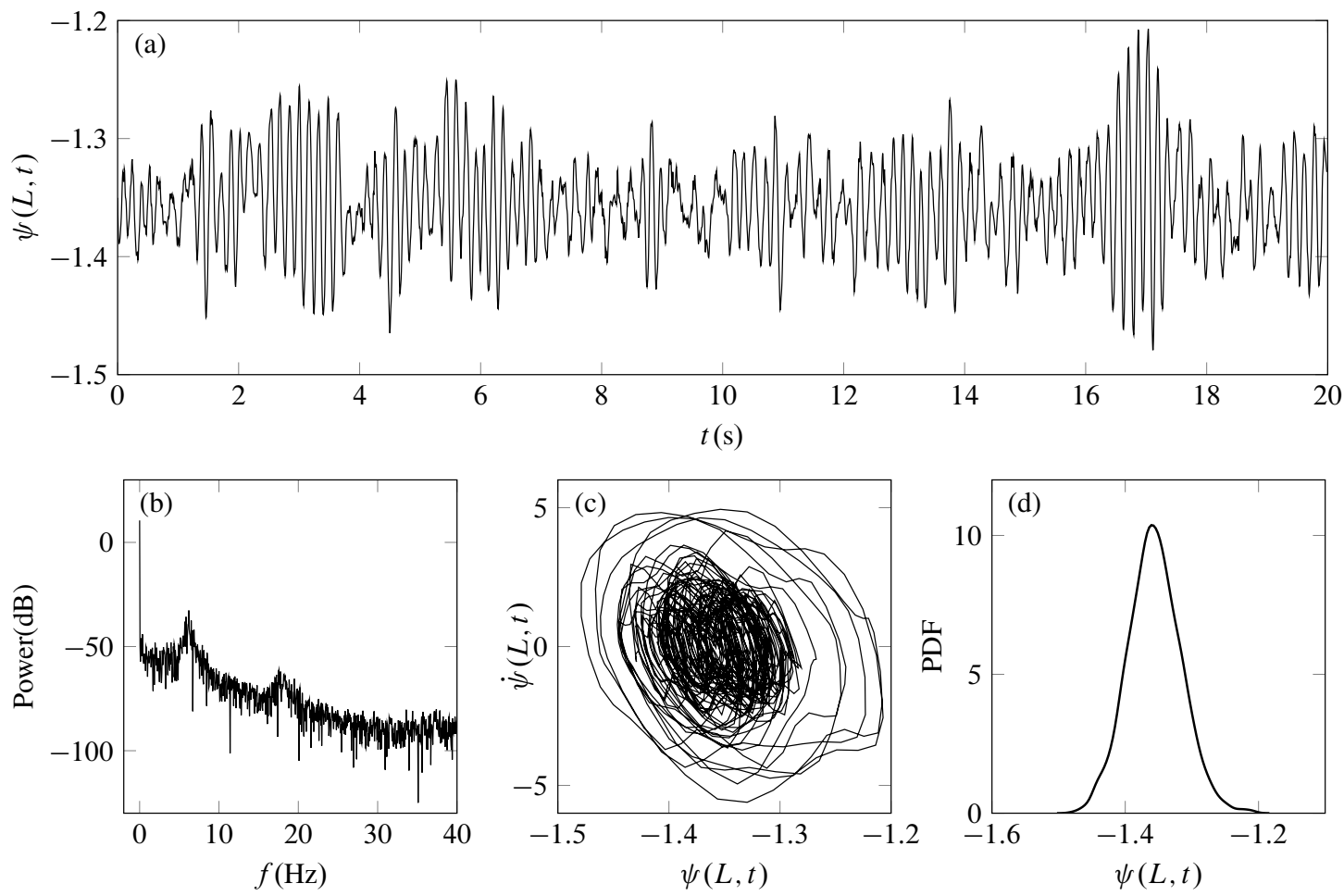

Figure 18: Experimental results at $U=14.5 \mathrm{~m} / \mathrm{s}(\Pi=2.88)$ for the flag of Figure 7 with a random motion around the deflected equilibrium; (a) time history, (b) PSD, (c) phase-plane portrait, and (d) PDF of the response.

\section{Comparison between theory and experiment}

The global qualitative dynamical behaviour (i.e. the sequence and types of bifurcations) of inverted flags predicted by the theoretical model was described in the previous sections of this paper, and it was found to be in fairly good agreement with experimental observations. Both theory and experiments show that the stability of large-aspect-ratio inverted flags is quite different from that of very-low-aspect-ratio (or slender) ones. Hysteresis in the critical values and sensitivity of the dynamics to imperfections (mainly, presence of initial curvature/angle of attack) are other facets of qualitative agreement between theory and experiments. Some of these comparisons are summarized in Table 4.

\begin{tabular}{lccc}
\hline & & $\begin{array}{c}\text { Qualitative route } \\
\text { (increasing } \Pi \text { ) }\end{array}$ & $\begin{array}{c}\text { Mechanism of the } \\
\text { first instability }\end{array}$ \\
\hline $\begin{array}{l}\text { Theory: Model I }(\$ 2.2) \\
\text { Theory: Model II }(\$ 2.3)\end{array}$ & $\begin{array}{c}\mathrm{U}<0.1 \\
\mathrm{ND}\end{array}$ & $\mathrm{UE} \rightarrow \mathrm{SAD} \rightarrow \mathrm{LAD}$ \\
\hline Experiments & $R<0.1$ & $\mathrm{UE} \rightarrow \mathrm{LAD} \rightarrow \mathrm{LAF}$ & $\begin{array}{c}\text { Subcritical pitchfork } \\
\text { Supercritical pitchfork }\end{array}$ \\
\hline & $0.1<R<4$ & $\mathrm{UE} \rightarrow \mathrm{SAD} \rightarrow \mathrm{SAF} \rightarrow \mathrm{LAF} \rightarrow \mathrm{C} \rightarrow \mathrm{LAD}$ & $\begin{array}{c}\text { Divergence } \\
\text { with large hysteresis } \\
\text { Divergence } \\
\text { with gradual bending }\end{array}$ \\
\hline
\end{tabular}

Table 4: Qualitative comparison between theoretical models and experiment with increasing flow speed. UE, undeformed equilibrium; SAD, small-amplitude static deformation; SAF, small-amplitude flapping; LAF, largeamplitude flapping; DF, deformed flapping; C, chaos; LAD, large-amplitude static deformation.

The numerical results from the nonlinear models for the dynamics of inverted flags discussed in Sections 2.2 and 2.3 are used for a deeper quantitative comparison with the experimental measurements. To do so, the models proposed by Tavallaeinejad et al. $(2018,2020 a, b)$ have been solved using parameters matching those of the inverted flags in the experiments.

For slender flags, the numerical results are validated against the measurements presented in Figure 23 for $R=0.1$ and $R=0.07$. In particular, the flow velocity at which the flags jump spontaneously to the large-amplitude deflected state with increasing flow velocity $\left(\Pi_{\mathrm{LAD}}^{+}\right.$in experiments and $\Pi_{\mathrm{P}}$ in theory) and the flow velocity at which the flags return to the undeflected equilibrium ( $\Pi_{\mathrm{LAD}}^{-}$in experiments and $\Pi_{\mathrm{S}}$ in theory) are compared in Table 5. For the theoretical results, two cases are considered for each aspect ratio: a perfectly straight inverted flag, and one with an initial tip deflection, $\Delta / L$. It is recalled that the subcritical pitchfork bifurcation (i.e. $\Pi_{\mathrm{P}}$ ) is replaced by a saddle-node bifurcation, $\Pi_{\mathrm{S} 1}$, in the presence of imperfections. 

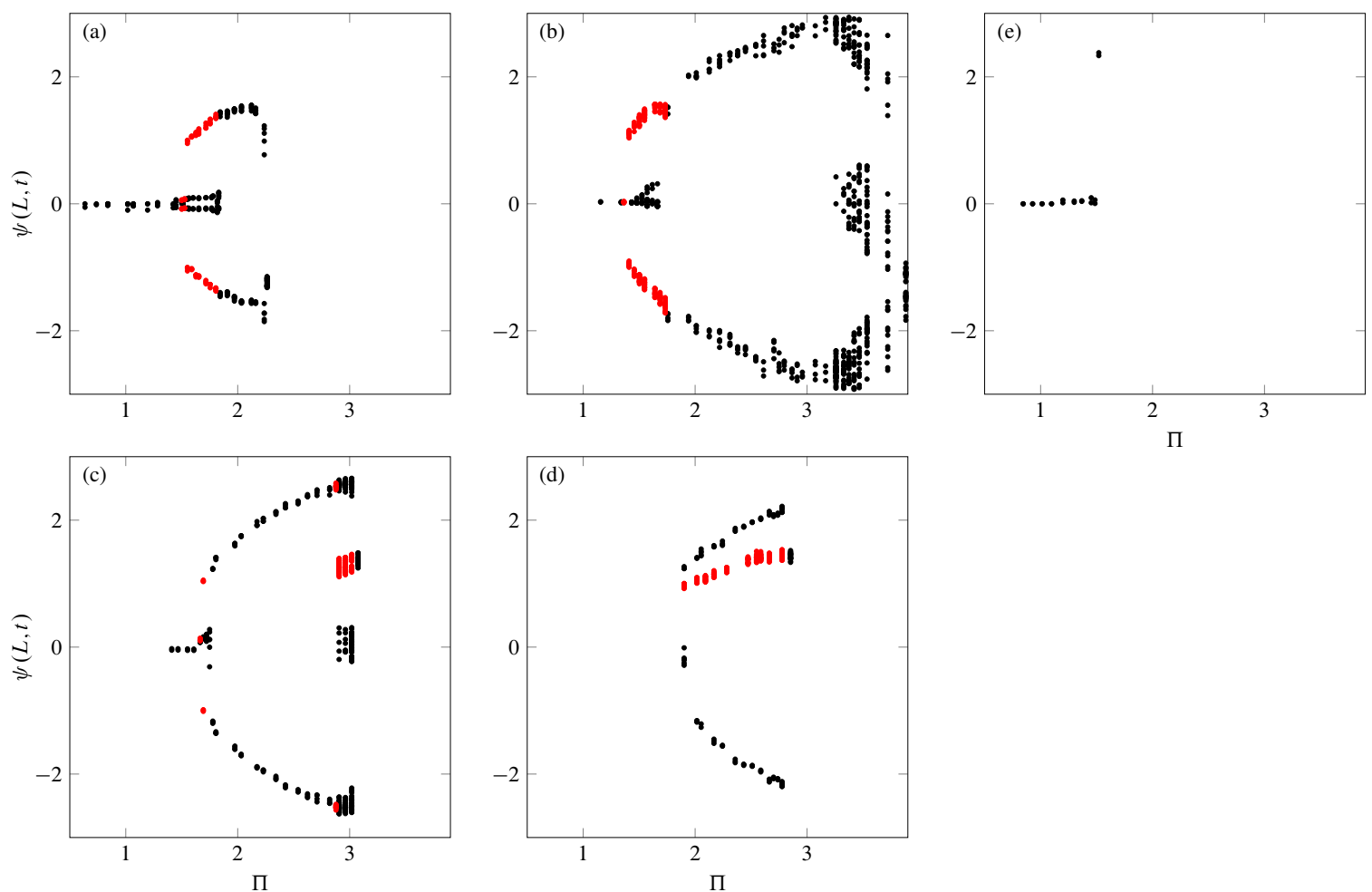

Figure 19: Bifurcation diagrams of inverted flags made of (a) brass with $\mu=0.07$, (b) stainless steel with $\mu=0.15$, (c) zinc with $\mu=0.20$, (d) polycarbonate with $\mu=0.25$, and (e) polypropylene with $\mu=0.33$. Black and red symbols correspond to wind-speed sweep up and down, respectively.

In Table 5, a good quantitative agreement can be seen between $\Pi_{S}$ (with or without the initial tip deflection) and $\Pi_{\mathrm{LAD}}^{-}$, although the experimental values, as expected, are lower than the theoretical ones. On the other

\begin{tabular}{c|ccc|ccc}
\hline Aspect ratio & Experiment & \multicolumn{2}{|c|}{ Theory $\Pi_{\mathrm{P}}\left(\Pi_{\mathrm{S} 1}\right)$} & Experiment & \multicolumn{2}{c}{ Theory $\Pi_{\mathrm{S}}$} \\
& $\Pi_{\mathrm{IAD}}^{+}$ & $\Delta / L=0$ & $\Delta / L=0.02$ & $\Pi_{\mathrm{LAD}}^{-}$ & $\Delta / L=0$ & $\Delta / L=0.02$ \\
\hline 0.07 & $4.3-4.5$ & 7.05 & 4.66 & $2.7-2.8$ & 2.98 & 2.96 \\
0.1 & $3.7-4.6$ & 5.81 & 4.20 & $2.6-3.3$ & 2.95 & 2.91 \\
\hline
\end{tabular}

Table 5: Comparison between experimental measurements presented in Figure 23 and the proposed model (Model I) for slender inverted flags. $\Pi_{\mathrm{LAD}}^{+}$: onset of large-amplitude deflection and $\Pi_{\mathrm{LAD}}^{-}$: the flow speed at which the flag returns to the undeflected equilibrium in the experiments; $\Pi_{\mathrm{P}}$ : onset of pitchfork bifurcation, $\Pi_{\mathrm{S} 1}$ : onset of the first saddle-node bifurcation for imperfect flags, and $\Pi_{S}$ : the saddle-node bifurcation in theory.

hand, the predicted values for the onset of divergence, $\Pi_{\mathrm{P}}$, overestimate markedly the measured ones in the experiment, $\Pi_{\mathrm{LAD}}^{+}$.

The discrepancy between the onset of divergence found experimentally and theoretically, $\Pi_{\mathrm{LAD}}^{+}$and $\Pi_{\mathrm{P}}$, respectively, is partially due to the presence of perturbations in the oncoming flow, leading to small-amplitude vibrations around the undeflected equilibrium. As predicted by the theoretical model, the basin of attraction for the undeflected equilibrium is very small over a wide range of the flow velocities. Hence, small perturbations can cause the flag to deflect at velocities smaller than that predicted by theory. In addition, as also predicted by the theory, the presence of an initial curvature along the flags reduces $\Pi_{\mathrm{P}}$. This is evident from the results obtained for cases with $\Delta / L=0.02$, as compared to $\Delta / L=0$. Thus, a seemingly small tip deflection significantly reduces the predicted critical value, resulting in better agreement between theory and experiments.

The dimensionless critical flow velocities found experimentally, $\Pi_{\mathrm{D}}, \Pi_{\mathrm{SAF}}^{+}, \Pi_{\mathrm{LAF}}^{+}$, and $\Pi_{\mathrm{LAF}}^{-}$, for instabilities of wide inverted flags ( $R=2.0, R=3.0$, and $R=4.0$ ) as well as the dimensionless frequency of oscillations at $\Pi_{\mathrm{LAF}}^{+}$(denoted by $f_{\mathrm{f}}^{*}$ ) are listed in Table 6 . Also shown are the theoretical values of critical flow velocities for a two-dimensional inverted flag, for comparison purposes.

It is seen that theoretical and experimental critical flow velocities agree reasonably well for $\psi_{0}=0$, although the theoretical values generally overestimate the measured ones. This is not surprising, upon considering the effect of inevitable imperfections in the experiments; as predicted by theory, a consequence of a small initial angle of attack is the reduction in the critical flow values. In the theoretical values obtained with $\psi_{0}=0.1$ also shown in the table, lower critical flow velocities are obtained, which, compared to those for $\psi_{0}=0$, are generally in better agreement with the experimental values. The measured dimensionless 

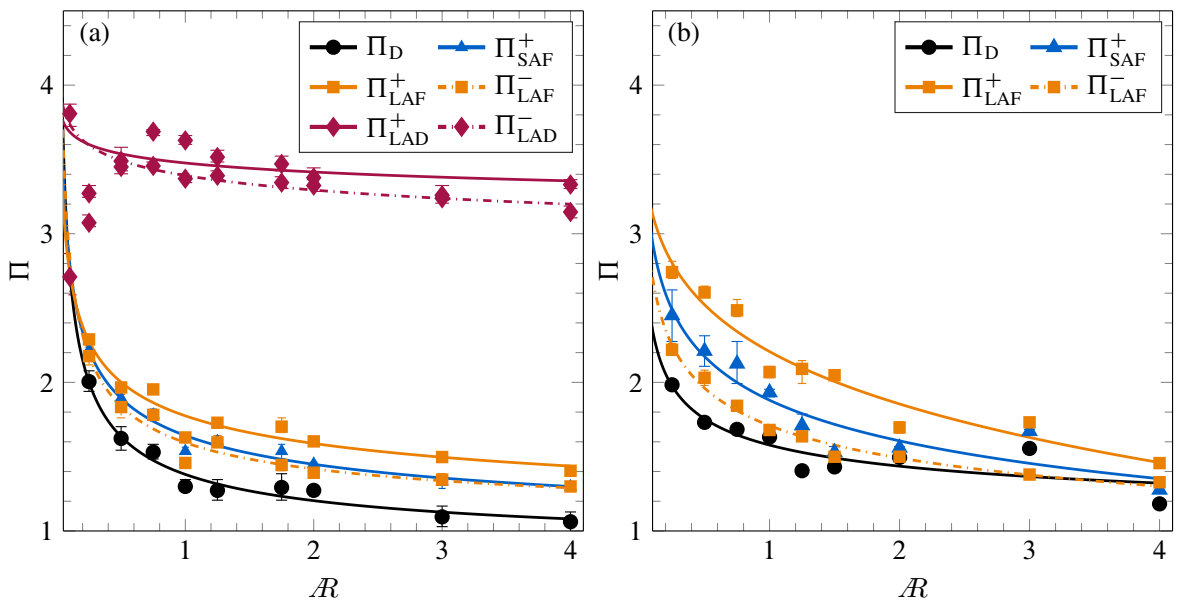

Figure 20: Stability boundaries for flags of similar mass ratio but different aspect ratios: (a) $\mu=0.2$; (b) $\mu=0.15$.
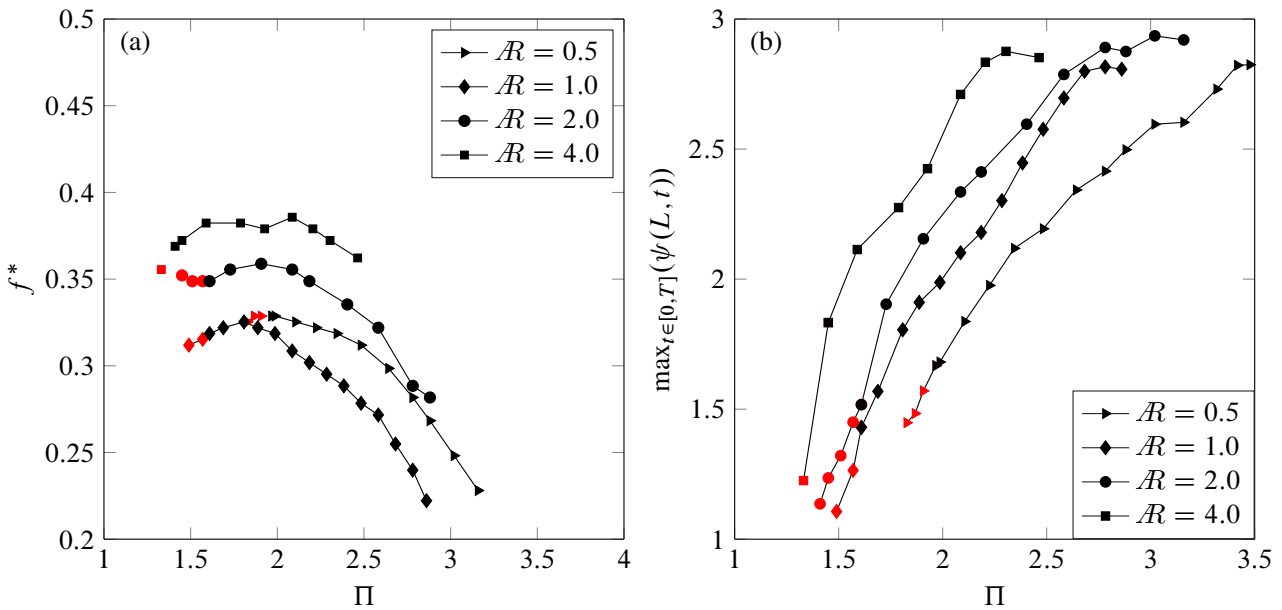

Figure 21: Dimensionless flapping (a) frequency $f^{*}=f \sqrt{\rho_{\mathrm{p}} A / D} L^{2}$ and (b) amplitude of inverted flags with different aspect ratios but the same mass ratio $\mu=0.20$.

frequencies at the onset of the large-amplitude flapping were found to be smaller than the numericallycomputed values; this may be attributed to limitations of the theoretical model in capturing the viscous effects well enough.

A remark should be made on the 2D assumption made in the theory. 2D flow theory may be considered accurate for very wide (or very-large-aspect-ratio) flags, although tip vortex and 3D effects are still dominant close to the side-edges. On the other hand, the 2D structural model based on the Euler-Bernoulli beam theory is more suitable for low and very-low-aspect-ratio flags (wider flags may exhibit large-amplitude spanwise bending and torsional displacements which are not considered in the 2D structural model). Combining the two conflicting requirements, it appears that the $2 \mathrm{D}$ theoretical model is in fact suitable for predicting the dynamics of finite but large aspect-ratio flags, such as those presented in Table 6.

Finally, Figure 24 shows the variation of the maximum amplitude in the large-amplitude flapping regime as a function of $\Pi$. The experimental results for different flags are drawn with different markers, whilst the theoretical results are shown as solid lines. A generally good agreement between theory and experiment can be observed.

\section{Conclusion}

Experiments were described in this paper, aimed mainly at examining the global dynamics of inverted flags for various system parameters. Experiments were conducted in a subsonic wind tunnel with a fairly large test-section and low turbulence level, allowing for reliable measurements. The flow velocity in the test-section was incremented in small steps, and the flag motion was recorded via a high-speed camera at each step. An image processing technique was then utilized to extract the time history of oscillations. Different analysis tools were used to characterize the flag motion and to provide insight into the underlying mechanisms.

Six different states/regimes were identified for large-aspect-ratio flags as the flow velocity was increased, namely (i) the stretched-straight state with small vibrations superimposed, (ii) the buckled state in the form of 

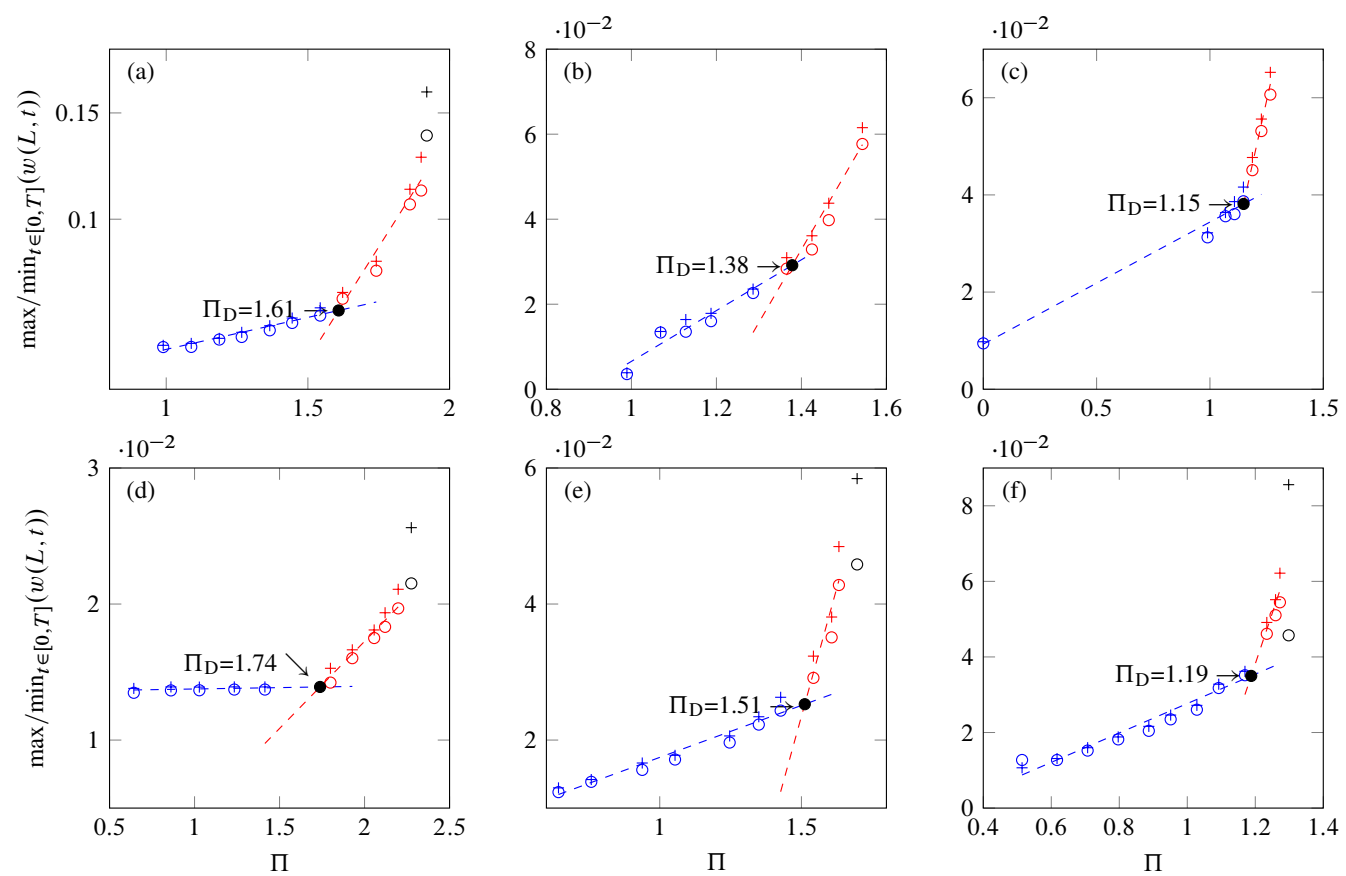

Figure 22: $[+]$ Maximum and $[\bigcirc]$ minimum dimensionless transverse displacement of the leading edge of the flag over a period of oscillations, as a function of increasing dimensionless flow velocity, indicating the development of divergence for several aspect ratios: (a) $R=0.5$, (b) $R=2.0$, (c) $R=4.0$ with $h=0.76 \mathrm{~mm}$; (d) $R=0.5$, (e) $R=2.0$, (f) $R=4.0$ with $h=1.02 \mathrm{~mm}$. Although in some cases the post-critical equilibrium was associated with a negative tip deflection, the absolute value of $w(L, t)$ is plotted in all subfigures for consistency.

\begin{tabular}{cccccc}
\hline & \multicolumn{3}{c}{ Experiment } & \multicolumn{2}{c}{ Theory $(2 \mathrm{D})$} \\
\cline { 2 - 6 }$R$ & 2.0 & 3.0 & 4.0 & $\psi_{0}=0$ & $\psi_{0}=0.1$ \\
\hline$\Pi_{\mathrm{D}}$ & $1.25-1.51$ & $1.03-1.56$ & $0.99-1.19$ & 1.36 & 1.03 \\
$\Pi_{\mathrm{SAF}}^{+}$ & $1.43-1.56$ & $1.29-1.67$ & $1.29-1.31$ & 1.71 & 1.54 \\
$\Pi_{\mathrm{LAF}}^{+}$ & $1.58-1.71$ & $1.49-1.74$ & $1.39-1.45$ & 1.85 & 1.84 \\
$\Pi_{\mathrm{LAF}}^{-}$ & $1.39-1.51$ & $1.31-1.39$ & $1.29-1.33$ & 1.80 & 1.79 \\
$f_{\mathrm{f}}^{*}$ & 0.35 & 0.35 & 0.37 & 0.56 & 0.56 \\
\hline
\end{tabular}

Table 6: Comparison between experimental measurements presented in Figure 20 and the proposed model in $\S 2.3$ for wide inverted flags with $\mu=0.2 . f_{\mathrm{f}}^{*}$ denotes the dimensionless frequency at the onset of large-amplitude flapping.

a small mean deflection with small-amplitude random-like vibrations superimposed, (iii) coherent, periodic small-amplitude oscillations around the deflected equilibrium, (iv) large-amplitude periodic flapping around the undeflected equilibrium, (v) aperiodic (chaotic) regime with intermittent flapping around the origin and to one side, and finally (vi) the fully deflected state with large-amplitude deflection. Moreover, bistability and hysteresis were present in the flow ranges between the straight and flapping regimes and between the flapping and deflected regimes.

For very-low-aspect-ratio or slender flags, on the other hand, the sequence was found to be very simple, namely from (i) directly to (vi).

The observed sequence of dynamical states for both very-low- and large-aspect-ratio flags was found to be in very good agreement with the theoretically predicted sequence.

Stability maps were presented for flags of different aspect ratios from the results obtained in the present experiments, which agree with the general trends predicted via the theoretical models. It was found that the dependence of critical flow velocities on the aspect ratio is strong for narrow (or very-low-aspect ratio) inverted flags, while it is relatively weak for wide (or large-aspect-ratio) ones. The critical flow velocity for the onset of flapping approaches a nearly constant value when the flag is sufficiently wide, signaling that the flow is essentially two-dimensional. It was also found that the critical flow velocity for the onset of the fully-deflected state is almost insensitive to $R$. Additionally, for inverted flags with $R \gtrsim 2$, it was found that the ratio of the critical flow velocities for large amplitude divergence and flapping, $\Pi_{\mathrm{LAD}} / \Pi_{\mathrm{LAF}} \approx 2$.

The hysteresis phenomenon, the existence of which was predicted by our nonlinear theoretical model, was clearly observed in the experiments with flags of various materials and aspect ratios. It was shown that, within the hysteresis loop, an inverted flag would remain at its undeflected equilibrium, whereas if subjected to a relatively large external disturbance, it would undergo self-excited oscillations at the same flow velocity. 


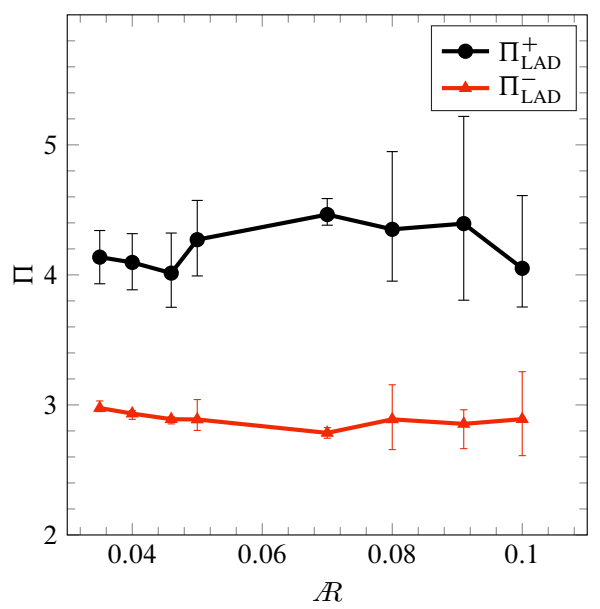

Figure 23: Stability boundaries for slender flags with different aspect ratios.

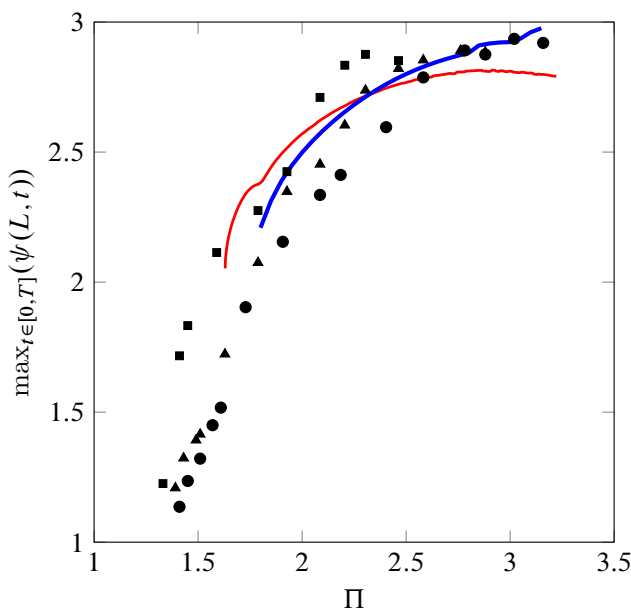

Figure 24: Experimental values for the maximum tip rotation angle for $[\boldsymbol{\Theta}] R=2.0,[\mathbf{\Delta}] R=3.0$, and [ $R=4.0$, versus those obtained via the analytical model of $\S 2.3$ : [ - ] perfectly straight $2 \mathrm{D}$ flag, $[-] 2 \mathrm{D}$ flag with $\psi_{0}=0.1$. In all cases, $\mu=0.20$.

The dynamics of inverted flags was also explored experimentally with flags with a wide variety, vis-à-vis previous experiments, of materials. Flags of different materials were found to display different quantitative and qualitative dynamics.

Quantitative agreement between experimental and theoretical critical flow velocities for different very-lowand large-aspect-ratio flags was found to be fairly good. Considering a slight initial tip deflection (to emulate geometric imperfections) resulted in even better agreement between theory and experiment. The experimental and theoretical results for the maximum rotation angle were also found to be comparable.

\section{Acknowledgments}

The authors gratefully acknowledge the financial support by the Natural Sciences and Engineering Research Council of Canada, the Solution Mining Research Institute (SMRI) and Pipeline Research Council International (PRCI). It is a pleasure to acknowledge the benefit of insightful comments from Professor John E. Sader, particularly on the topics of Section 3. Thanks also go to Professor Guy Gauthier of ÉTS.

\section{References}

BOLLAY, W. 1939 A non-linear wing theory and its application to rectangular wings of small aspect ratio. ZAMM-Journal of Applied Mathematics and Mechanics 19 (1), 21-35.

BuCHAK, P., ELOY, C. \& REIS, P. M. 2010 The clapping book: wind-driven oscillations in a stack of elastic sheets. Physical Review Letters 105 (19), 194301.

Chen, Y., Yu, Y., Zhou, W., Peng, D. \& LiU, Y. 2018 Heat transfer enhancement of turbulent channel flow using tandem self-oscillating inverted flags. Physics of Fluids 30 (7), 075108. 
Cossé, J., SAder, J., Kim, D., Cerdeira, C. H. \& Gharib, M. 2014 The effect of aspect ratio and angle of attack on the transition regions of the inverted flag instability. In ASME 2014 Pressure Vessels and Piping Conference. Paper PVP2014-28445.

COSSÉ, J. T. 2014 On the behavior of pliable plate dynamics in wind: application to vertical axis wind turbines. PhD thesis, California Institute of Technology, Pasadena, CA, USA.

Eloy, C., Kofman, N. \& Schouveiler, L. 2012 The origin of hysteresis in the flag instability. Journal of Fluid Mechanics 691, 583-593.

FAn, B., Huertas-Cerdeira, C., Cossé, J., SAder, J. E. \& Gharib, M. 2019 Effect of morphology on the large-amplitude flapping dynamics of an inverted flag in a uniform flow. Journal of Fluid Mechanics 874, 526-547.

GozA, A., Colonius, T. \& SAder, J. E. 2018 Global modes and nonlinear analysis of inverted-flag flapping. Journal of Fluid Mechanics 857, 312-344.

GUNASEKARAN, S., Ross, G. V. \& CURRY, D. 2019 Effect of flexible inverted pvdf in free shear layer wake. In AIAA Scitech 2019 Forum, p. 1338.

Gurugubelli, P. \& JAiman, R. 2019 Large amplitude flapping of an inverted elastic foil in uniform flow with spanwise periodicity. Journal of Fluids and Structures 90, 139-163.

Gurugubelli, P. S. \& Jaiman, R. K. 2015 Self-induced flapping dynamics of a flexible inverted foil in a uniform flow. Journal of Fluid Mechanics 781, 657-694.

Kim, D., Cossé, J., Huertas Cerdeira, C. \& GHARIB, M. 2013 Flapping dynamics of an inverted flag. Journal of Fluid Mechanics 736.

Koreanschi, A., Sugar-Gabor, O. \& BoteZ, R. M. 2016 Numerical and experimental validation of a morphed wing geometry using Price-Païdoussis wind-tunnel testing. The Aeronautical Journal 120 (1227), 757-795.

LEE, J. B., PARK, S. G. \& Sung, H. J. 2018 Heat transfer enhancement by asymmetrically clamped flexible flags in a channel flow. International Journal of Heat and Mass Transfer 116, 1003-1015.

LI, K., WANG, S., KE, Z. \& CHEN, C.-L. 2019 A novel caudal-fin-inspired hourglass-shaped self-agitator for air-side heat transfer enhancement in plate-fin heat exchanger. Energy Conversion and Management 187, $297-315$.

Lighthill, M. J. 1971 Large-amplitude elongated-body theory of fish locomotion. Proceedings of the Royal Society of London B 179 (1055), 125-138.

Michelin, S. \& DoARÉ, O. 2013 Energy harvesting efficiency of piezoelectric flags in axial flows. Journal of Fluid Mechanics 714, 489-504.

Mosbah, A. B., Salinas, M. F., Botez, R. \& DaO, T.-M. 2013 New methodology for wind tunnel calibration using neural networks-EGD approach. SAE International Journal of Aerospace 6 (2013-01-2285), 761-766.

Murugesan PAZHANi, K. \& ACHARYA, S. 2019 An experimental investigation of the dynamics of an inverted serrated flag. In AIAA Scitech 2019 Forum, pp. 1891(1-17).

Orrego, S., Shoele, K., Ruas, A., Doran, K., Caggiano, B., Mittal, R. \& Kang, S. H. 2017 Harvesting ambient wind energy with an inverted piezoelectric flag. Applied Energy 194, 212-222.

PAÏDOUSSIS, M. P. 2016 Fluid-Structure Interactions: Slender Structures and Axial Flow. Volume 2, 2nd edition. Oxford: Academic Press.

Païdoussis, M. P., Grinevich, E., AdAmovic, D. \& Semler, C. 2002 Linear and nonlinear dynamics of cantilevered cylinders in axial flow. Part 1: Physical dynamics. Journal of Fluids and Structures 16 (6), 691-713.

PARK, S. G., Kim, B., ChAng, C. B., RYU, J. \& Sung, H. J. 2016 Enhancement of heat transfer by a self-oscillating inverted flag in a poiseuille channel flow. International Journal of Heat and Mass Transfer 96, 362-370.

Polhamus, E. C. 1966 A concept of the vortex lift of sharp-edge delta wings based on a leading-edge-suction analogy. NASA Technical Note, TN D-3767 .

RinALDI, S. \& PAÏDOUSSIS, M. P. 2012 Theory and experiments on the dynamics of a free-clamped cylinder in confined axial air-flow. Journal of Fluids and Structures 28, 167-179.

RinALDI, S. \& PAÏDOUSSIS, M. P. 2020 An improved theoretical model for the dynamics of a free-clamped cylinder in axial flow. Journal of Fluids and Structures 94, 102903.

RYU, J., PARK, S. G., KIM, B. \& SUNG, H. J. 2015 Flapping dynamics of an inverted flag in a uniform flow. Journal of Fluids and Structures 57, 159-169.

SAder, J. E., Cossé, J., KIM, D., FAn, B. \& GHARIB, M. 2016 a Large-amplitude flapping of an inverted flag in a uniform steady flow - a vortex-induced vibration. Journal of Fluid Mechanics 793, 524-555.

SAdER, J. E., HuERTAS-CERdeirA, C. \& GHARIB, M. 2016 $b$ Stability of slender inverted flags and rods in uniform steady flow. Journal of Fluid Mechanics 809, 873-894.

Shoele, K. \& Mittal, R. 2016 Flutter instability of a thin flexible plate in a channel. Journal of Fluid Mechanics 786, 29-46. 
Tavallaeinejad, M., Legrand, M. \& PAÏDoussis, M. P. $2020 a$ Nonlinear dynamics of slender inverted flags in uniform steady flows. Journal of Sound and Vibration 467, 115048.

TAVAllaeinejad, M., PAÏDoussis, M. P. \& LEgRAnd, M. 2018 Nonlinear static response of low-aspectratio inverted flags subjected to a steady flow. Journal of Fluids and Structures 83, 413-428.

Tavallaeinejad, M., Païdoussis, M. P., Legrand, M. \& Kheiri, M. $2020 b$ Instability and the post-critical behaviour of two-dimensional inverted flags in axial flow. Journal of Fluid Mechanics 890, A14.

Tavallaeinejad, M., Païdoussis, M. P., Salinas, M. F. , Legrand, M., Kheiri, M. \& Botez, R. M. 2020c Flapping of heavyinverted flags: a fluidelastic instability. Journal of Fluid Mechanics Rapids 904, R5-1.

THEODORSEN, T. 1949 General theory of aerodynamic instability and the mechanism of flutter. NACA Technical Report, No. 496 .

Yu, Y., LiU, Y. \& AmAndolese, X. 2019 A review on fluid-induced flag vibrations. Applied Mechanics Reviews 71 (1), 010801.

YU, Y., LIU, Y. \& CHEN, Y. 2017 Vortex dynamics behind a self-oscillating inverted flag placed in a channel flow: Time-resolved particle image velocimetry measurements. Physics of Fluids 29 (12), 125104(1-15). 